\title{
Atmospheric losses of radiation belt electrons
}

\author{
R. S. Selesnick and J. B. Blake \\ The Aerospace Corporation, Los Angeles, California, USA \\ R. A. Mewaldt \\ California Institute of Technology, Pasadena, California, USA \\ Received 23 July 2003; revised 23 September 2003; accepted 6 October 2003; published 31 December 2003.
}

[1] A numerical model of the low-altitude energetic electron radiation belt, including the effects of pitch angle diffusion into the atmosphere and azimuthal drift, predicts lifetimes and longitude-dependent loss rates as a function of electron energy and diffusion coefficient. It is constrained by high-altitude $(\sim 20,000 \mathrm{~km})$ satellite measurements of the energy spectra and pitch angle distributions and then fit to low-altitude $(\sim 600 \mathrm{~km})$ data that are sensitive to the longitude dependence of the electron losses. The fits provide estimates of the parameterized diffusion coefficient. The results show that the simple driftdiffusion model can account for the main features of the low-altitude radiation belt inside the plasmasphere during periods of steady decay. The rate of pitch angle diffusion is usually stronger on the dayside than on the nightside, frequently by a factor $\sim 10$. The average derived lifetimes for loss into the atmosphere of $\sim 10$ days are comparable to the observed trapped electron decay rates. Considerable variability in the loss rates is positively correlated with geomagnetic activity. The results are generally consistent with electron scattering by plasmaspheric hiss as the primary mechanism for pitch angle diffusion. INDEX TERMS: 2716 Magnetospheric Physics: Energetic particles, precipitating; 2720 Magnetospheric Physics: Energetic particles, trapped; 2730 Magnetospheric Physics: Magnetosphere-inner; 2753 Magnetospheric Physics: Numerical modeling; KEYWORDS: electrons, radiation belt, atmospheric losses, precipitation

Citation: Selesnick, R. S., J. B. Blake, and R. A. Mewaldt, Atmospheric losses of radiation belt electrons, J. Geophys. Res., 108(A12), 1468, doi:10.1029/2003JA010160, 2003.

\section{Introduction}

[2] The substantial role of pitch angle diffusion as a mechanism for radiation belt electron loss into the atmosphere has been qualitatively demonstrated by low-altitude satellite data [Imhof, 1968; Sheldon, 1991]. However, limitations in spatial coverage and the problem of determining which electrons subsequently will be lost make it difficult to directly measure the loss rate. Observations of trapped electron decay rates [Williams et al., 1968; Roberts, 1969; Pesnell et al., 2001] provide an indirect estimate but can be influenced by other processes such as radial diffusion. Theoretical calculations of pitch angle diffusion based on scattering by plasma waves and Coulomb collisions [Lyons et al., 1972; Abel and Thorne, 1998] are in reasonable agreement with the observed decay rates, but a direct determination of the electron losses probably requires a combination of the theoretical and experimental approaches. Fortunately, the nondipolar component of the geomagnetic field (or, equivalently, the offset of the dipole from the center of the Earth) causes losses to occur nonuniformly in longitude, and the resulting redistribution of electrons by azimuthal drift provides the necessary observational constraints for theoretical

Copyright 2003 by the American Geophysical Union. 0148-0227/03/2003JA010160\$09.00 modeling of the atmospheric losses. The validity of this approach to modeling the observed low altitude electron distribution was demonstrated by Imhof [1968], based on an approximate analytic solution to the diffusion equation. A similar concept with a simplified construction was described by Sheldon [1991]. Detailed simulations by Abel and Thorne [1999] accounted for both diffusion and drift but were not compared directly with electron data. These works suggested that a simple balance between azimuthal drift and pitch angle diffusion can represent the low-altitude electron distribution. Our goal in this work is to further explore the validity of this idea using an extensive satellite electron data set. Time periods of steady electron decay and locations inside the plasmasphere but near the peak intensity of the outer radiation belt are chosen for study. We start by describing a parameterized theoretical model, then apply constraints from high-altitude electron data taken on the Polar satellite to simulate observations from the low-altitude SAMPEX satellite, and finally obtain estimates of the diffusion coefficients and associated loss rates by determining the model parameters that best fit the data.

\section{Drift-Diffusion Model}

[3] A simplified description of radiation belt electron dynamics above the upper atmosphere is 

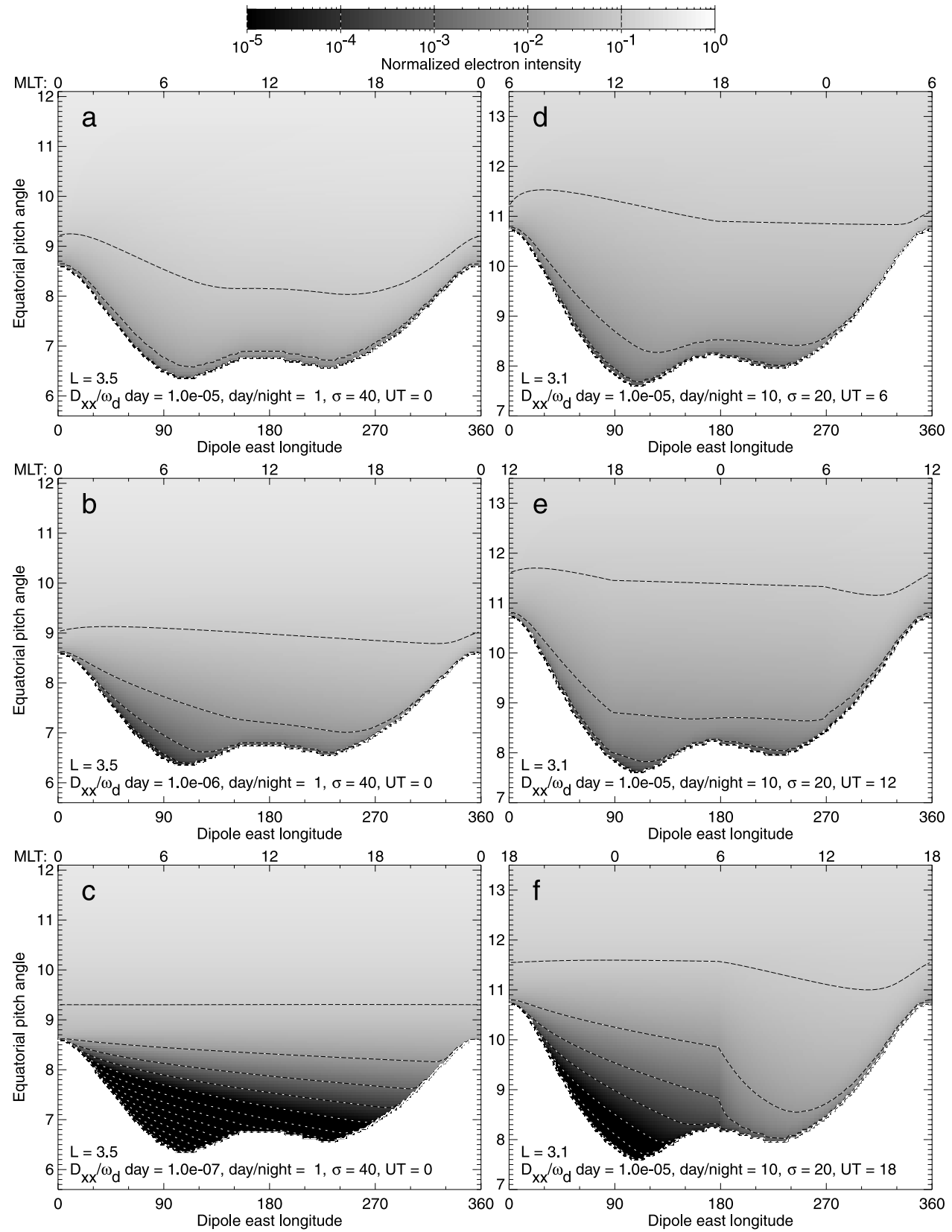

Figure 1. Color-coded solutions of the model drift-diffusion equation with selected values of the model parameters. They are normalized to $f=1$ at $\alpha_{0}=90^{\circ}$. The lower boundary where $f=0$ is the bounce loss cone angle. Dashed contours are shown every decade in $f$. Figures $1 \mathrm{a}, 1 \mathrm{~b}$, and $1 \mathrm{c}$ have equal day and night diffusion coefficients but decreasing rates of diffusion relative to azimuthal drift. Figures 1d, 1e, and $1 \mathrm{f}$ all have the same diffusion coefficient, with the daytime value 10 times greater than the nighttime one, but are for different universal times (UT). The $L$ shells and $\sigma$ values also differ $\left(D_{x x} \sim x^{-\sigma}\right)$. See color version of this figure in the HTML.

$$
\frac{\partial f}{\partial t}+\omega_{d} \frac{\partial f}{\partial \phi}=\frac{1}{x T(y)} \frac{\partial}{\partial x}\left(x T(y) D_{x x} \frac{\partial f}{\partial x}\right),
$$

where the left-hand side is an advective time derivative including the effects of azimuthal drift and the right-hand side is the rate of pitch angle diffusion. The equation models the electron phase space density $f(x, \phi, t)$ that is averaged over the phases of the gyration and bounce motions, but it retains the dependence on the drift phase or azimuth $\phi$. It also depends on time $t$ and $x=\cos \alpha_{0}$, where $\alpha_{0}$ is the equatorial pitch angle. The $x$ diffusion coefficient due to pitch angle scattering is $D_{x x}$. For the diffusion term [Schulz and Lanzerotti, 1974, p. 56], $T$ is proportional to the electron bounce period and we approximate to a dipole magnetic field with [Davidson, 1976] $T(y)=1.380173-0.639693 y^{0.737}$, where $y=\sin$ $\alpha_{0}$. The electron energy determines the drift frequency $\omega_{d}$, which we also approximate as being independent of $\phi$ as in a dipole field. 
[4] Electron losses are included in the model by the lowaltitude boundary condition, for which we assume $f=0$ at the edge of the bounce loss cone (BLC). (High or low altitude is equivalent to high or low $\alpha_{0}$ and to low or high $x$ ). We define the BLC as the range of equatorial pitch angles where an electron's adiabatic mirror point reaches below an altitude of $100 \mathrm{~km}$ in either hemisphere. The nondipolar nature of the Earth's magnetic field at low altitudes causes the BLC angle for a given drift shell to vary with $\phi$. For the high altitude boundary condition we require $d f f f x=0$ at $x=0\left(\alpha_{0}=90^{\circ}\right)$.

[5] The nature of the solutions to the model equation (1) is that the details of any initial condition are rapidly lost, after which a characteristic dependence of $f$ on $x$ and $\phi$ is obtained and decays away exponentially in time at a rate determined by the diffusion coefficient $D_{x x}$. The characteristic shape during the exponential decay phase is also the lowest order eigenmode of the combined drift-diffusion operator in equation (1), with the corresponding eigenvalue representing the decay rate. Higher-order eigenmodes may be included in the initial condition but have faster decay rates. The lowest-order eigenmode is a function of $x$ and $\phi$ that depends only on the dimensionless ratio $D_{x x} / \omega_{d}$. Some examples are shown in Figure 1. They were obtained by numerically solving equation (1) by a finite-differencing technique for a time sufficiently long to reach the exponential decay phase and then normalizing each solution by its value at the high altitude boundary $\alpha_{0}=90^{\circ}$. The lowaltitude boundary is the bounce loss cone angle for a given $L$ shell value, calculated from the IGRF magnetic field model [Barton, 1997]. The azimuthal angle $\phi$ is the dipole east longitude which increases in the same direction as the electron drift and is measured relative to the Earth's dipole axis, starting from the intersection of the dipole equator with the geographic prime meridian (thus it is nearly equivalent to geographic east longitude). The solutions are shown for only a small range of $x$ near the BLC. At lower $x$ values (higher mirror point altitudes) the $\phi$ dependence is insignificant (the solutions in this region are described in the next section).

[6] The variation with longitude of the BLC angle that forms the lower boundary of the model is determined primarily by the South Atlantic anomaly (SAA). It is the region of weak magnetic field that for these fixed $L$ values is centered near $\phi=0^{\circ}$ and causes the significant peak in the BLC angle at that location.

[7] The choice of a functional dependence for $D_{x x}$ on $x$ and $\phi$ offers a great variety of possible solutions. Guided by observations as detailed in the following sections, we have chosen the parameterized form

$$
D_{x x}=D_{\text {day } / n i g h t} E^{-\mu} \frac{1}{\epsilon+x^{\sigma}},
$$

where the four free parameters are $D_{\text {day }}, D_{\text {night }}, \mu$, and $\sigma$. The electron kinetic energy in $\mathrm{MeV}$ is $E$. Independent values of $D_{\text {day }}$ and $D_{\text {night }}$ are allowed for daytime and nighttime, respectively, as determined by the magnetic local time (MLT). The day-night, or MLT, dependence is translated into a corresponding dipole longitude $(\phi)$ dependence based on a given universal time (UT). A fixed value of $\epsilon=10^{-4}$ is included only for numerical stability

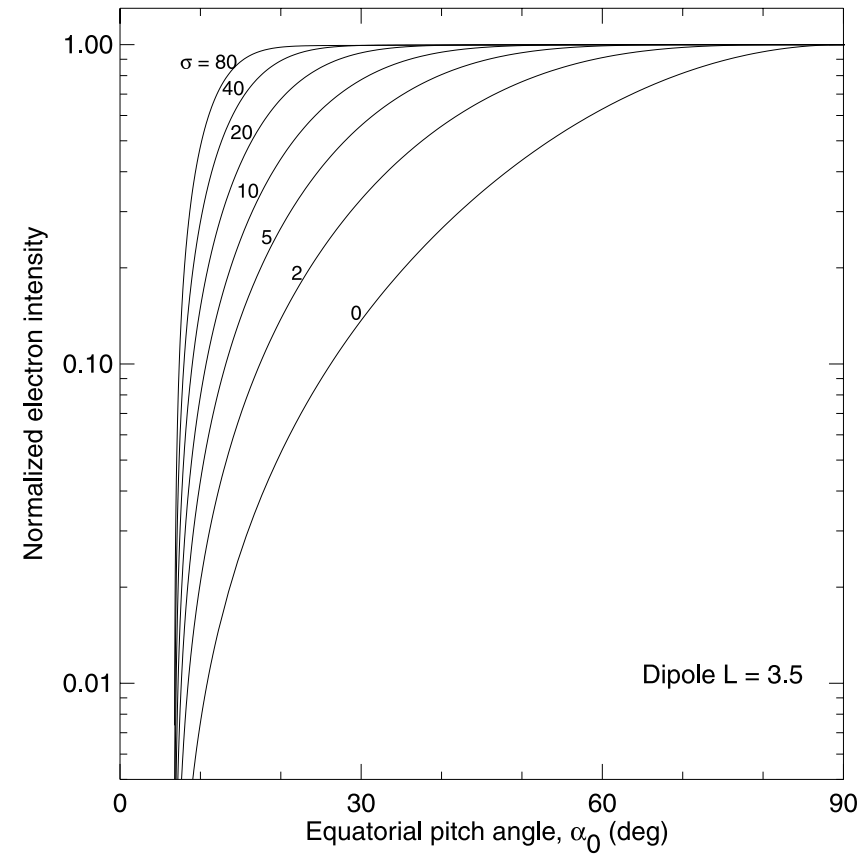

Figure 2. Model pitch angle distributions for selected $\sigma$ values $\left(D_{x x} \sim x^{-\sigma}\right)$ assuming a dipole magnetic field at $L=3.5$.

reasons. It prevents $D_{x x}$ from becoming too large at low $x$ values without significantly impacting the final solution. In Figure 1 the values of $D_{x x} / \omega_{d}$ refer to $x \approx 1$ (the region shown in the figure) and to the dayside, that is $D_{d a y} E^{-\mu} / \omega_{d}$. Note that the energy dependence $E^{-\mu}$ is included in this ratio but it is not significant for the individual model solutions. Its significance will become apparent when the solutions are combined for modeling observations over a range of electron energies.

[8] Referring to Figure 1, the three solutions on the lefthand side (Figures 1a, 1b, and 1c) have equal day and night values of $D_{x x}($ day/night $=1)$ but the ratio $D_{x x} / \omega_{d}$ decreases by a factor of 10 in each case. In the first case (Figure 1a), $D_{x x} / \omega_{d}$ has the high value of $10^{-5}$ and diffusion is dominant over drift. The solution reflects the $\phi$ dependence of the BLC angle, although there is a small influence of the drift as shown by the small eastward shift of the higher altitude contours. As the ratio $D_{x x} / \omega_{d}$ decreases (Figures $1 \mathrm{~b}$ and $1 \mathrm{c}$ ), drift becomes increasingly important relative to diffusion. This is shown by the contours becoming independent of $\phi$ in the stable trapping region, that is the region of $\alpha_{0}>8.5^{\circ}$, the highest BLC angle for $L=3.5$, and by the relative lack of electrons in the drift loss cone (DLC) to the east of the SAA. The DLC is the range of $\alpha_{0}$ values where electrons are not in the BLC but cannot complete a full drift without entering the BLC at another longitude. The DLC is relatively empty when $D_{x x} / \omega_{d}$ is small because the diffusion is too slow to fill it before drift takes the electrons toward the SAA where they are lost. At the low value of the ratio $D_{x x} / \omega_{d}=10^{-8}$ (Figure 1c) the $f$ value decreases by several orders of magnitude with decreasing $\alpha_{0}$ in the DLC. These three cases show that if diffusion is dominant then losses into the atmosphere are distributed evenly in longitude but that as drift become more significant, the losses, though still 


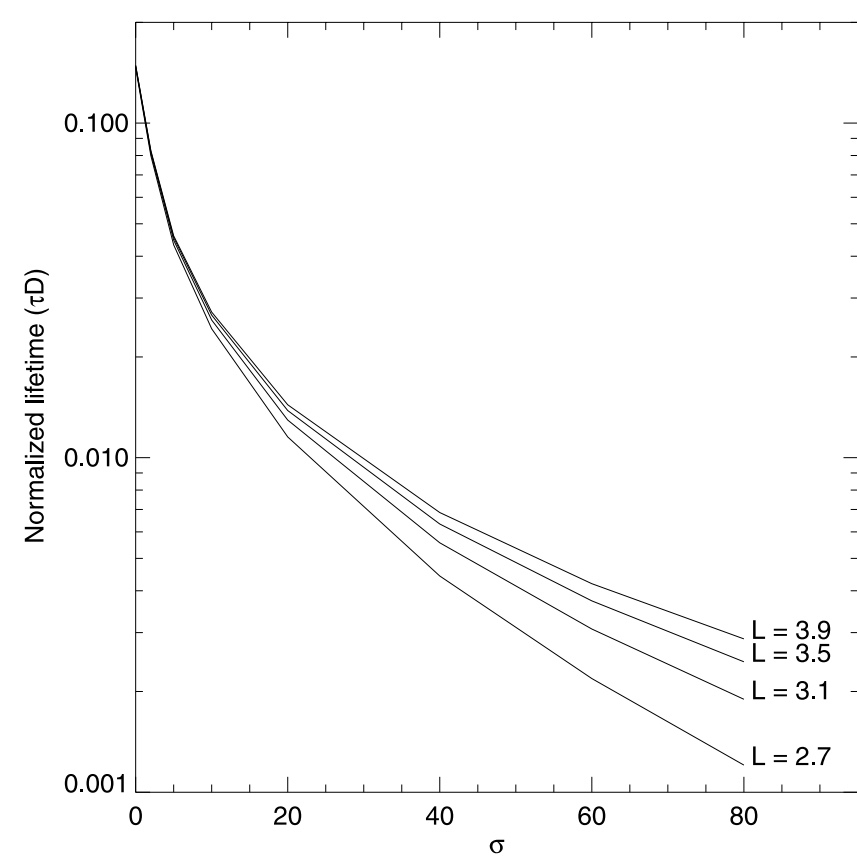

Figure 3. Normalized $e$-folding lifetime $\tau D$ versus $\sigma$, where $\tau$ is the lifetime and $D_{x x}=D x^{-\sigma}$, for selected $L$ values.

caused by diffusion, occur over a decreasing range of longitudes to the west of the SAA.

[9] The three solutions on the right-hand side of Figure 1 (Figures $1 \mathrm{~d}, 1 \mathrm{e}$, and 1f) all have a dayside value of $D_{x x} / \omega_{d}=$ $10^{-5}$ and a nightside value that is 10 times smaller (day/ night $=10)$. They differ only in the UT hours of the simulation which are (Figure 1d) 6, (Figure 1e) 12, and (Figure 1f) 18. Now diffusion is dominant on the dayside while drift is dominant on the nightside, as can be seen from the MLT (magnetic local time) labels in each case. In the first two (Figures 1d and 1e) the DLC region to the east of the SAA ( 0 to $90^{\circ}$ dipole longitude) is on the dayside and is filled in by diffusion. Then the eastward drift fills in the rest of the drift loss cone so that these solutions are similar to the case (Figure 1a) in which diffusion was dominant throughout. In the third case (Figure 1f) the DLC region to the east of the SAA is on the nightside where diffusion is weak and is therefore relatively empty, while to the east of $180^{\circ}$ dipole longitude the DLC is on the dayside and is filled in by diffusion. There is no possibility, at any UT hour, for the eastern half $\left(>180^{\circ}\right)$ of the DLC to be relatively empty compared with the western half $\left(<180^{\circ}\right)$ because the eastern half is always filled in either by diffusion from lower $x$ or by drift from the west.

[10] The examples of Figure 1 also illustrate the variation in the BLC angles with $L$ and the variation in the model solution with the $\sigma$ parameter. The first three cases (Figures 1a, 1b, and 1c) are for $L=3.5$ and $\sigma=40$. The next three cases (Figures 1d, 1e, and 1f) are for $L=3.1$ and $\sigma=20$.

\section{Model Lifetimes}

[11] The decay lifetimes of the model solutions, or equivalently the eigenvalues associated with the lowestorder eigenmodes, depend primarily on $D_{x x}$ and are nearly independent of $\omega_{d}$. This is because the BLC angles for a given $L$ vary over only a small range of $x$ and the lifetimes are therefore well approximated by those that would be obtained with an average value of the BLC angle, independent of $\phi$. In the case that $D_{x x}$ is also independent of $\phi$ we can drop the $\phi$ dependence of the solution altogether and solve a simpler equation in $x$ only. These solutions are illustrated in Figure 2. It shows the normalized pitch angle distributions for various values of $\sigma$, where $D_{x x}=D /(\epsilon+$ $\left.x^{-\sigma}\right)$ has the same $x$ dependence as before. The BLC angle is that for $L=3.5$ in a dipole magnetic field.

[12] The increasing slope of the pitch angle distributions near the BLC angle with increasing $\sigma$ suggests an inverse dependence of the model lifetimes on $\sigma$. This is illustrated in Figure 3, which shows the normalized $e$-folding lifetimes $\tau D$, where $\tau$ is the lifetime, versus $\sigma$ for various dipole $L$ values. (If the approximation $T=1$ is made in equation (1), then the one-dimensional eigenmodes and eigenvalues can be obtained analytically [Roberts, 1969]. They are similar to the numerical results obtained here.)

[13] In the case where $D_{x x}$ has a day/night asymmetry the one-dimensional solutions (Figure 2) and the normalized lifetimes (Figure 3) are still good approximations for $x$ values other than those near the BLC because they are independent of $D$. The lifetimes $\tau$ are then simply obtained

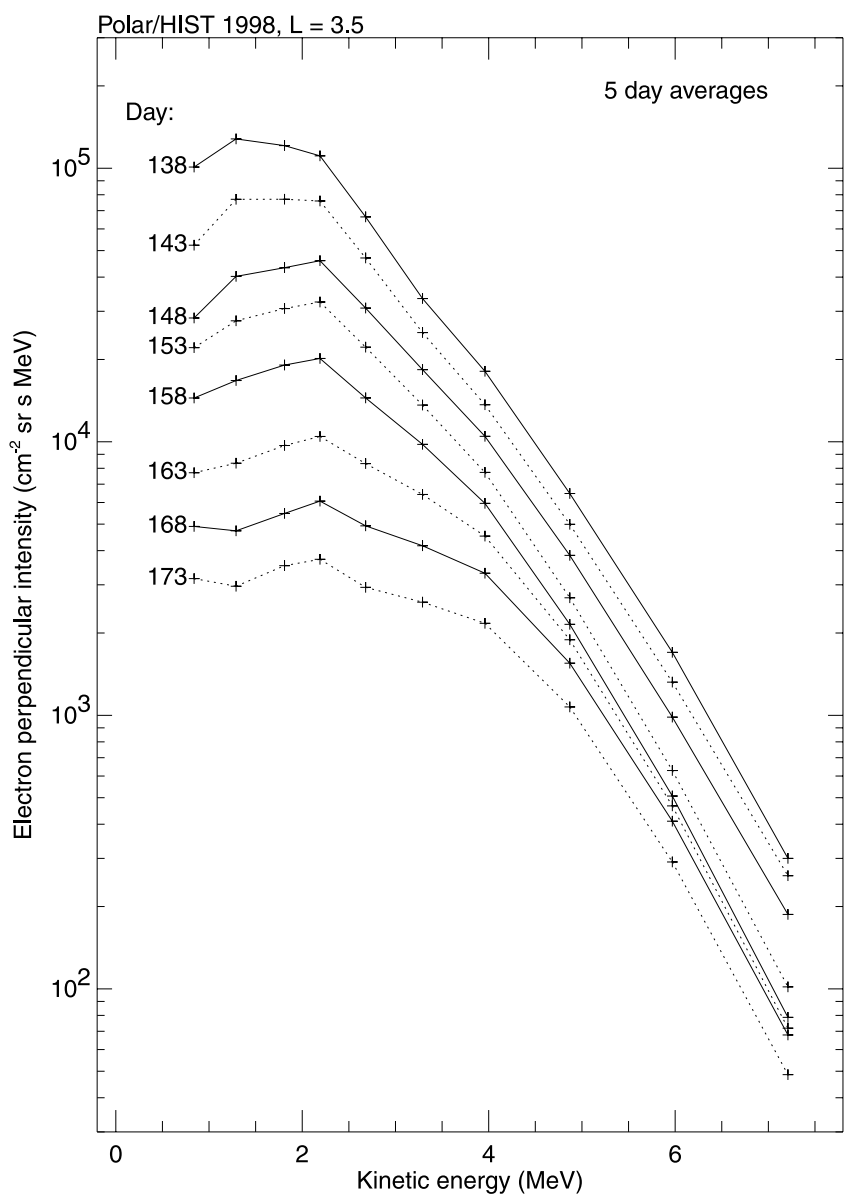

Figure 4. Electron energy spectra measured at high altitude on the Polar satellite, averaged over 5-day intervals centered on the labeled day numbers. 

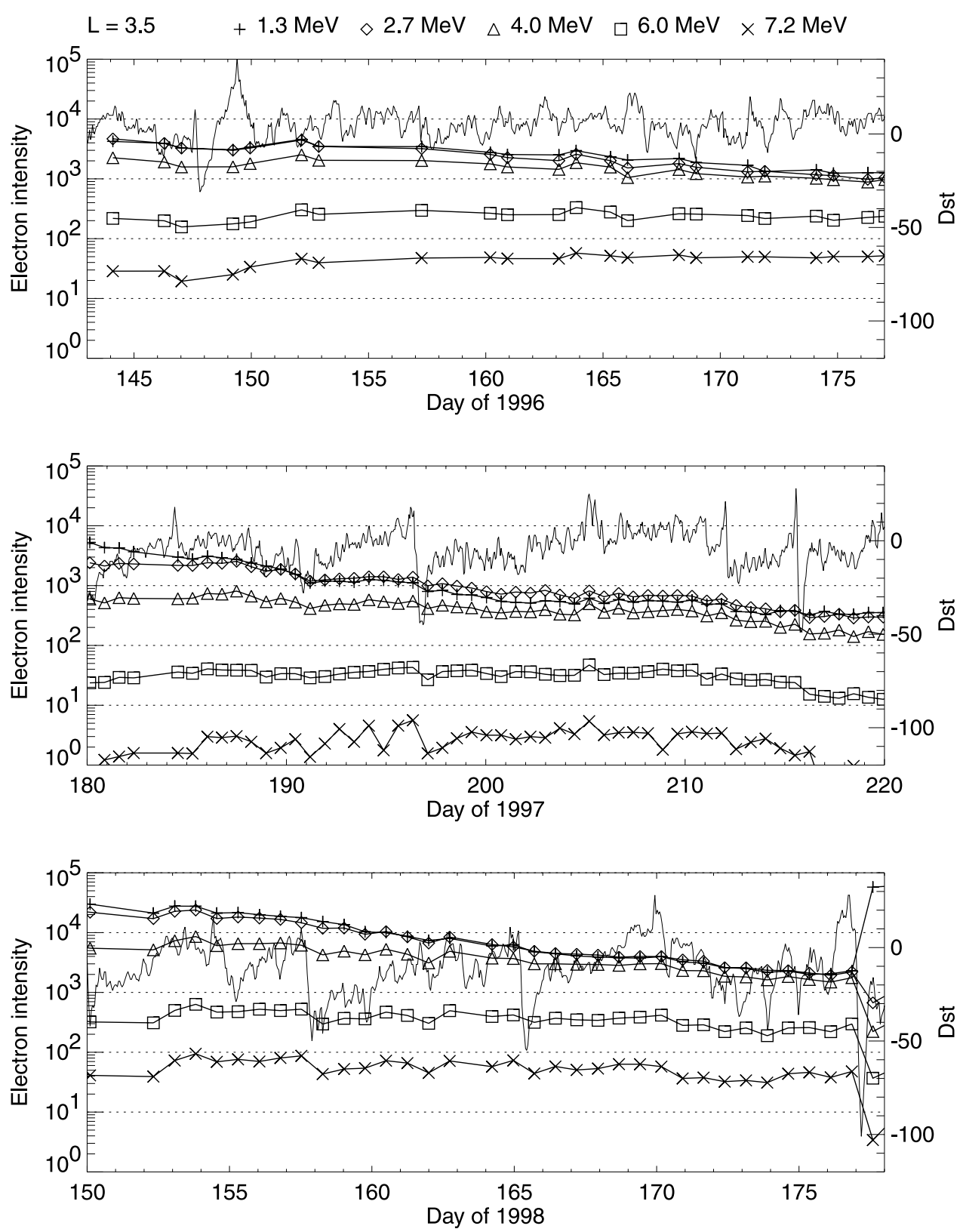

Figure 5. Electron intensity versus time for three selected time intervals, measured at $\alpha_{0}=40^{\circ}$ and at the indicated energies, from the Polar satellite (units as in Figure 4). The $D_{s t}$ geomagnetic index is also shown.

from the normalized lifetimes after division by the mean of the day and night $D$ values.

\section{High-Altitude Data}

[14] At high altitudes, where equatorial pitch angles $\alpha_{0}$ are well away from the loss cones, phase mixing by azimuthal drift causes the electron intensity to be independent of drift phase. Therefore measurements made at a single longitude can be used to normalize the model for simulating low-altitude data. Electron energy spectra from $L=3.5$ measured at high altitude on the Polar satellite are shown in Figure 4. They were taken during a 40-day period in 1998 following an electron injection $\sim 10$ days earlier. The data were obtained by HIST (the High Sensitivity
Telescope) [Blake et al., 1995] and analyzed using techniques described previously [Selesnick and Blake, 2000]. During this period the altitude of the elliptical Polar orbit at $L=3.5$ was $\sim 20,000 \mathrm{~km}$. We also use similar data from other $L$ shells.

[15] We have also chosen two other time periods, from mid-1996 and mid-1997, with similar characteristics of electron decay. The measured electron intensity at $L=3.5$ from all three periods, for selected energies, is shown versus time in Figure 5. It demonstrates the generally steady decay at the lower energies and relatively constant levels at the higher energies.

[16] The periods of generally decreasing intensity illustrated in Figure 5 were chosen so that the pitch angle distributions (PADs) are likely to have reached the expo- 


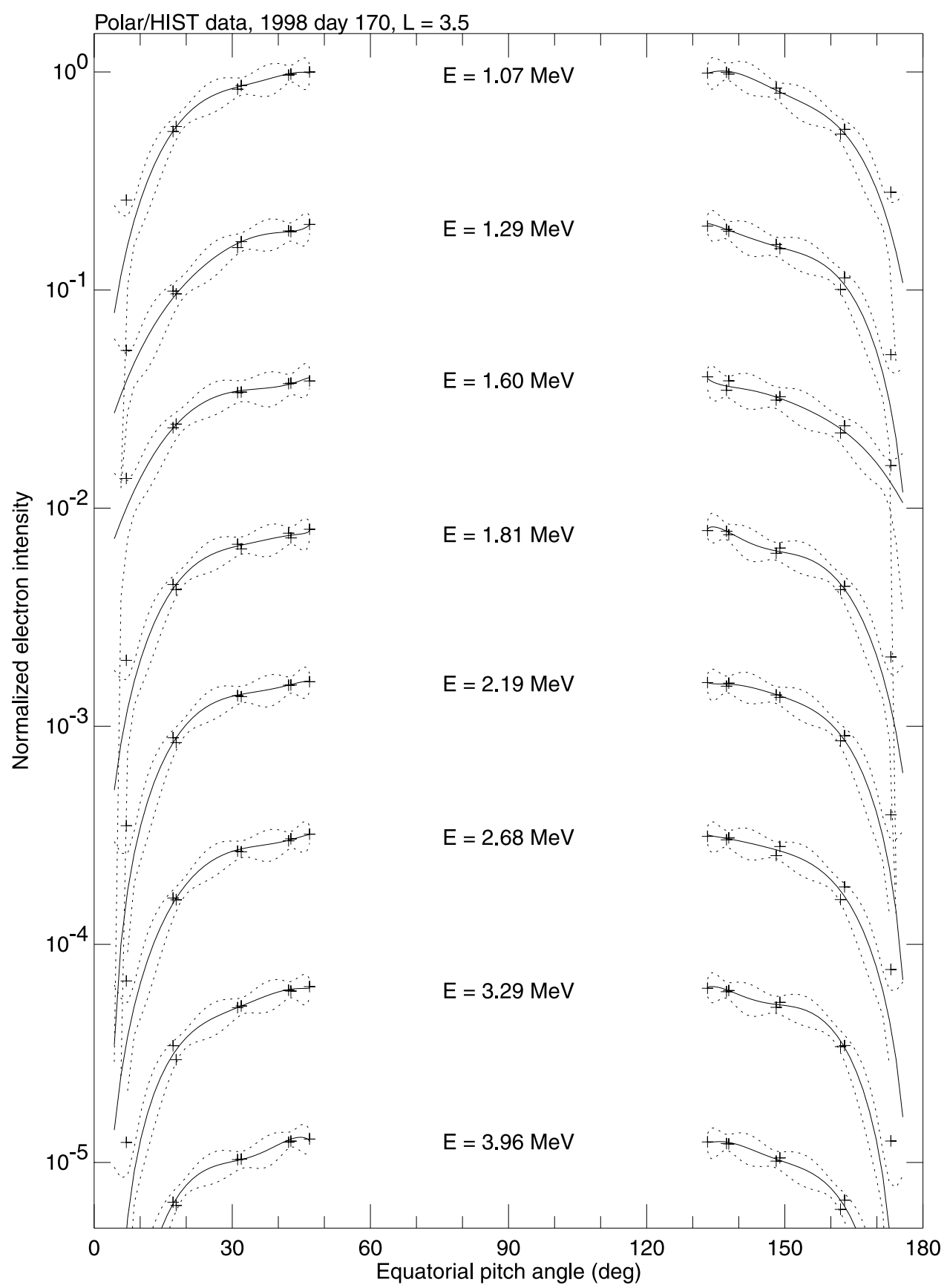

Figure 6. Electron equatorial pitch angle distributions measured simultaneously at the labeled energies from the Polar satellite, with normalization factors chosen for clarity of display. The smooth curves, with dashed uncertainty ranges, are the pitch angle distributions after deconvolution from the instrumental angular response and the satellite rotation sector width [Selesnick and Blake, 2000]. Data points are centered on each sector.

nential decay phase discussed earlier. Examples of the PADs measured by HIST near the end of the 1998 time interval are shown in Figure 6 for several electron energies. The shapes of these distributions are generally independent of energy, and measurements from earlier times show that they are also generally independent of time during the decaying period.

[17] The Polar data are used to normalize the model at high altitudes. The Polar altitude at the time of the measurement was such that equatorial pitch angles between $\alpha_{0} \approx 50^{\circ}$ and $130^{\circ}$ were not observable. Therefore the normalization is done at $\alpha_{0}=40^{\circ}$. This is easily sufficient for the distributions to be independent of drift phase. Measurements from other time periods show that the distributions at the angles that are unobserved in this case are essentially flat [e.g., Selesnick and Blake 1998], consistent with the high $\sigma$ model distributions (Figure 2).

[18] The measured PADs are not well resolved near the loss cone angles, $\alpha_{0} \lesssim 20^{\circ}$ and $\alpha_{0} \gtrsim 160^{\circ}$, so we cannot expect an accurate comparison with the model distributions 


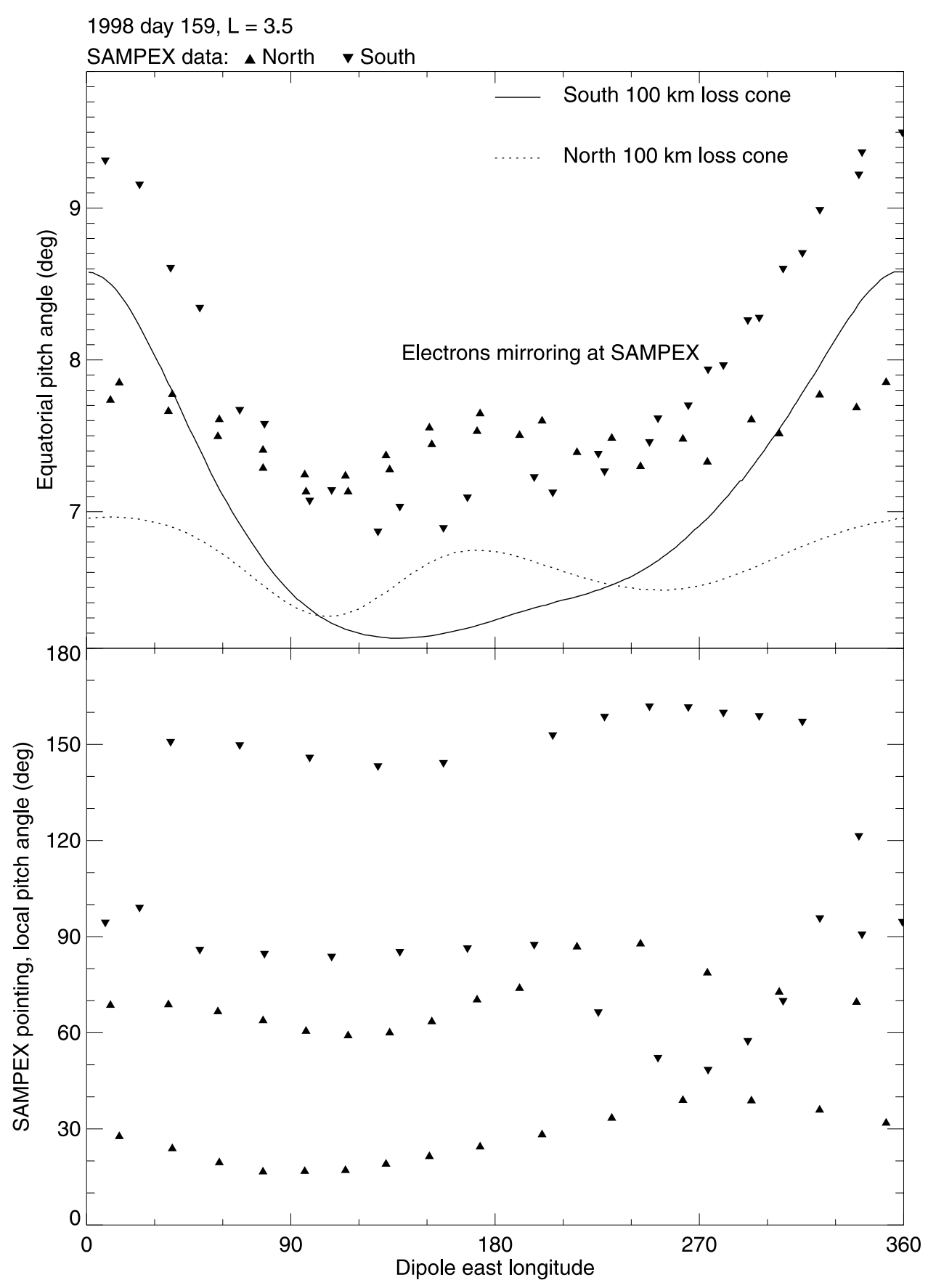

Figure 7. (top) Equatorial pitch angles as a function of longitude for electrons mirroring at the lowaltitude SAMPEX satellite. Each orbital crossing of $L=3.5$ in the Northern or Southern Hemisphere during a 1-day period is indicated, with the loss cones angles for each hemisphere. (bottom) Local pitch angles of the SAMPEX (and PET instrument) pointing directions at the same locations.

in those ranges. Low-altitude data are necessary for such comparisons.

\section{Low-Altitude Data}

[19] Once the model is normalized by the high-altitude data, it can be used to simulate a particular set of lowaltitude electron data. We use data taken by PET (the Proton/Electron Telescope) on the SAMPEX satellite [Cook et al., 1993]. Its high-inclination, low-altitude $(\sim 600 \mathrm{~km})$ orbit crosses each radiation belt $L$ shell approximately 60 times per day covering all longitudes and providing a suitable data set for comparison with the model results, as illustrated in Figure 7 for $L=3.5$.

[20] The equatorial pitch angles of electrons mirroring at SAMPEX (upper part of Figure 7) are a little above the BLC angles at the same longitude in each hemisphere. However, PET does not measure only the mirroring electrons (or the pitch angle distribution) but a weighted average of electrons mirroring at and below SAMPEX that depends 


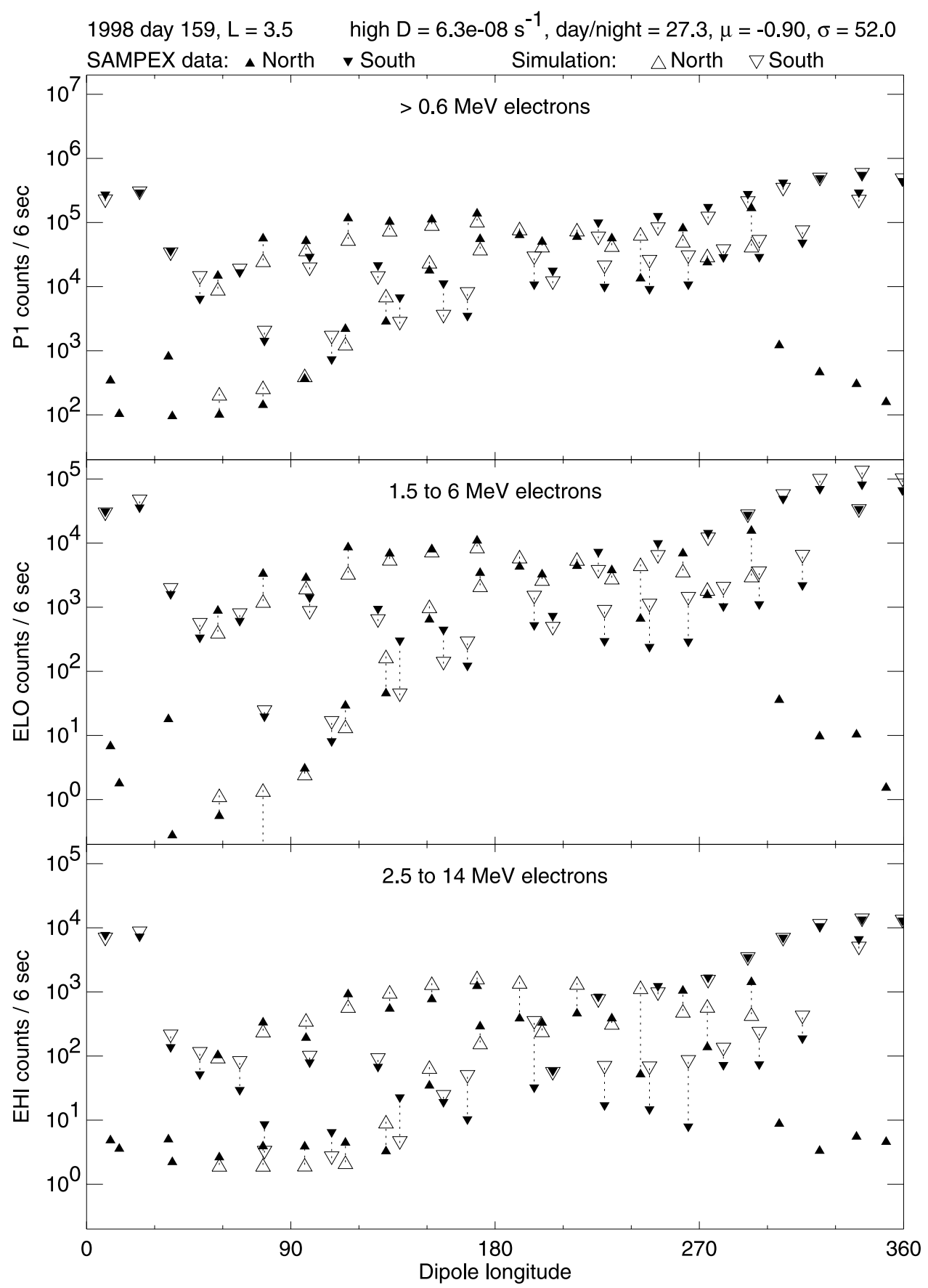

Figure 8. SAMPEX/PET electron data and model simulations for 1998 day 159. The data points are $6 \mathrm{~s}$ rate accumulations, interpolated to $L=3.5$, from the three rate counters, P1, ELO, and EHI, that respond to electrons from the approximate indicated energy ranges. The calibrated energy and angular response functions (see Appendix A) were used in the simulations. Dashed lines connect each data point to its corresponding simulated value. The model parameters were chosen to give the best fit to the data and included a high day/night diffusion ratio.

on the satellite pointing direction relative to local magnetic field. The variations in the pointing direction due to the four separate crossings of $L=3.5$ per orbit cover a wide range of local pitch angles (lower part of Figure 7). During the 1998 period of days 150 to 170 the local times of the SAMPEX orbit were within approximately 1 hour of the the noonmidnight plane. The satellite was rotating at 1 revolution per orbit, hence the regular pattern of pointing directions.
During the 1996 and 1997 periods it was rotating at 1 revolution per minute, providing a more random distribution of pointing directions at a given $L$ shell for those periods.

[21] We consider data taken by three PET electron rate counters, labeled P1, ELO, and EHI, that measure a low, medium, and high range of electron energies, respectively, together covering the range from $\sim 0.5$ to $14 \mathrm{MeV}$. 


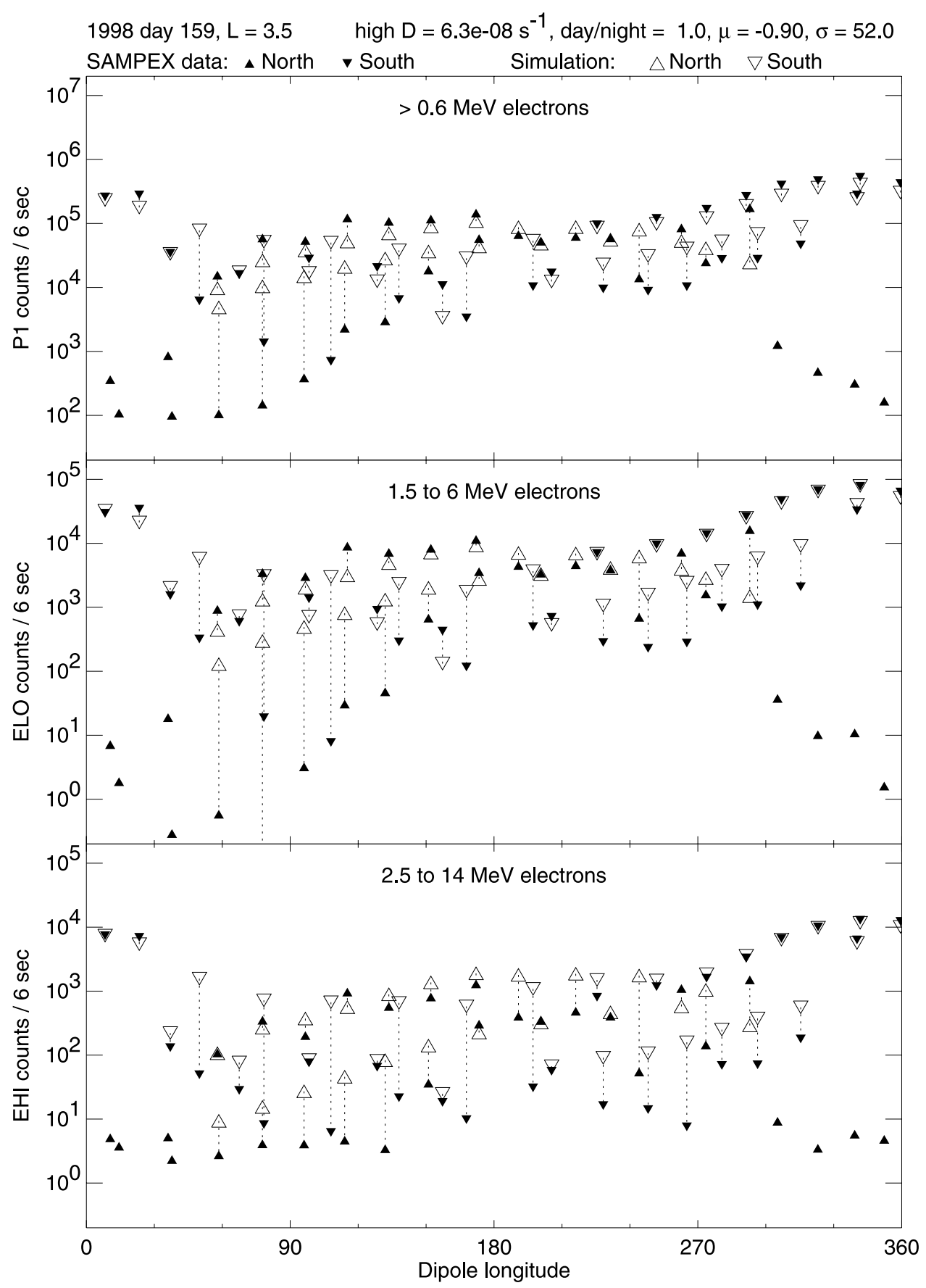

Figure 9. Similar to Figure 8 except that the nighttime diffusion coefficient was set equal to the daytime value from the best fit.

Samples of the data are shown in the next section. The flux (counting rate) from each counter is related to the electron intensity $j\left(E, \alpha_{0}\right)$ at each SAMPEX location by response functions that are described in Appendix A. These take into account the energy and angular response of each counter for a given pointing direction and are combined with the model results to simulate the counting rate at a given position on the orbit. The model is parametrized by the ratio $D_{x x} / \omega_{d}$, so the energy $E$ is determined for a given value of the diffusion coefficient $D_{x x}$ from the drift frequency $\omega_{d}$. Therefore a range of model solutions, corresponding to the appropriate range of energies, enters into the simulation of each rate counter.

\section{Low-Altitude Simulations}

[22] To provide strong constraints on the model parameters, the low-altitude data must sample the full range of longitudes and UT hours, so a full day of data is required. A sample set of data and the corresponding model simulation from 1998 day $159, L=3.5$ (the data corresponding to Figure 7) is shown in Figure 8. All of the PET flux measurements from the three rate counters (interpolated to 


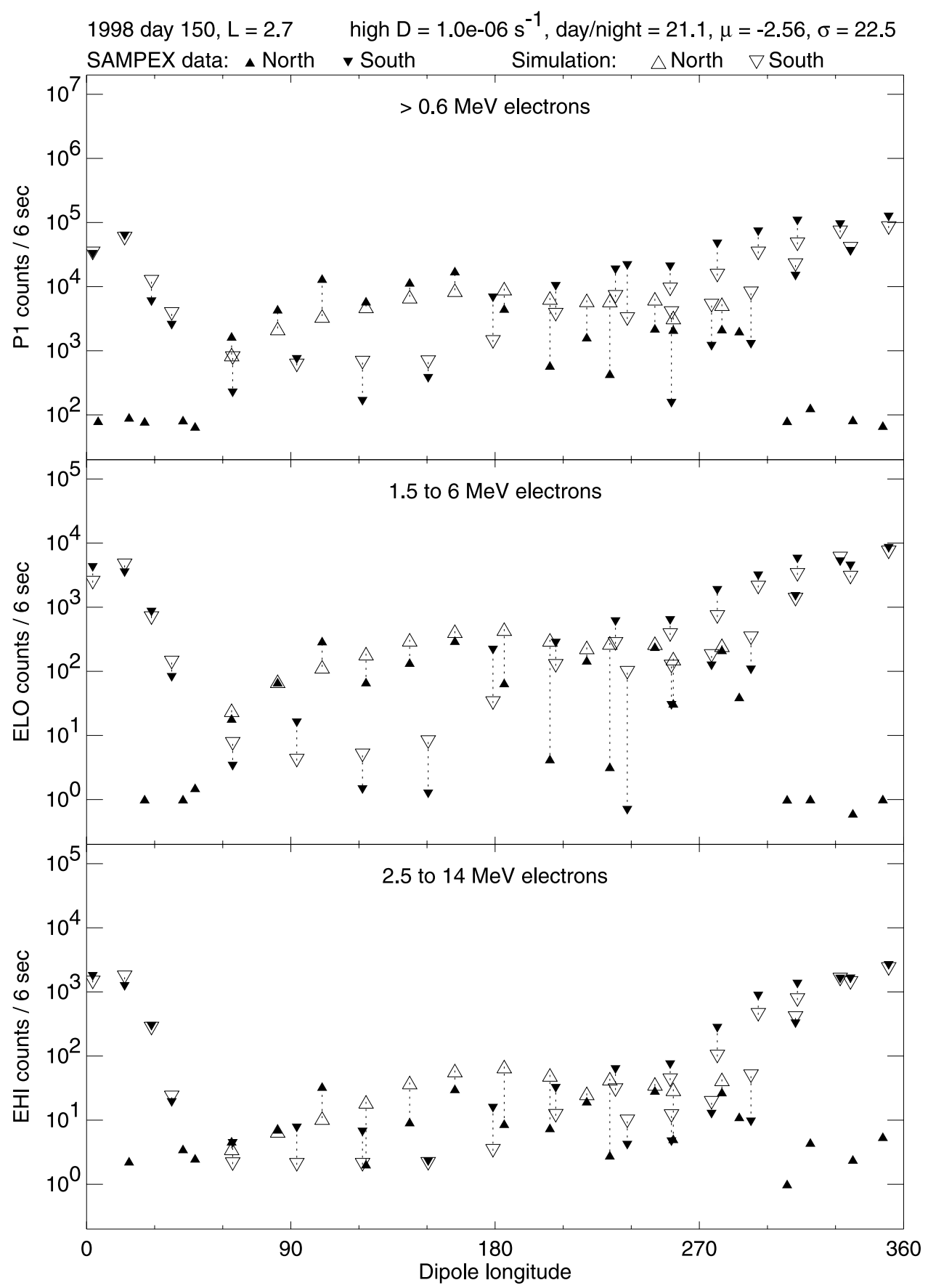

Figure 10. Similar to Figure 8 but for 1998 day 150 and $L=2.7$.

$L=3.5$ ) are shown, as a function of dipole longitude as in the previous figures, by the filled data points. The open points show the simulation that is the best fit to the data. The variations with longitude can be understood with reference to Figure 7. High fluxes from the Southern Hemisphere in the vicinity of the SAA $\left(\sim 0\right.$ and $360^{\circ}$ dipole east longitude) are stably trapped electrons that are primarily at equatorial pitch angles above the highest BLC angle. Low fluxes in the same longitude range but from the Northern Hemisphere are electrons in the BLC. They are generally $\lesssim 1 \%$ of the stably trapped Southern Hemisphere fluxes because, with backscattering from the atmosphere, electrons in the BLC can remain trapped for at most a few bounces. The model does not include any electrons in the BLC, so there are no simulations corresponding to the data in that region. Low fluxes to the east of the SAA (dipole east longitude $z 60^{\circ}$ ), from both north and south, gradually increase with longitude as the DLC is filled in by diffusion. On this date, the dayside diffusion coefficient was significantly ( $\sim 30$ times) greater than that on the nightside. Therefore during the first half of the day the western part of the DLC region $\lesssim 180^{\circ}$ ) showed substantially higher fluxes than during the second half of the day (compare Figures $1 \mathrm{~d}$ and $1 \mathrm{f}$ ). The day-night asymmetry accounts for most of the large range of observed and model fluxes at a given longitude in that region, although substantial 


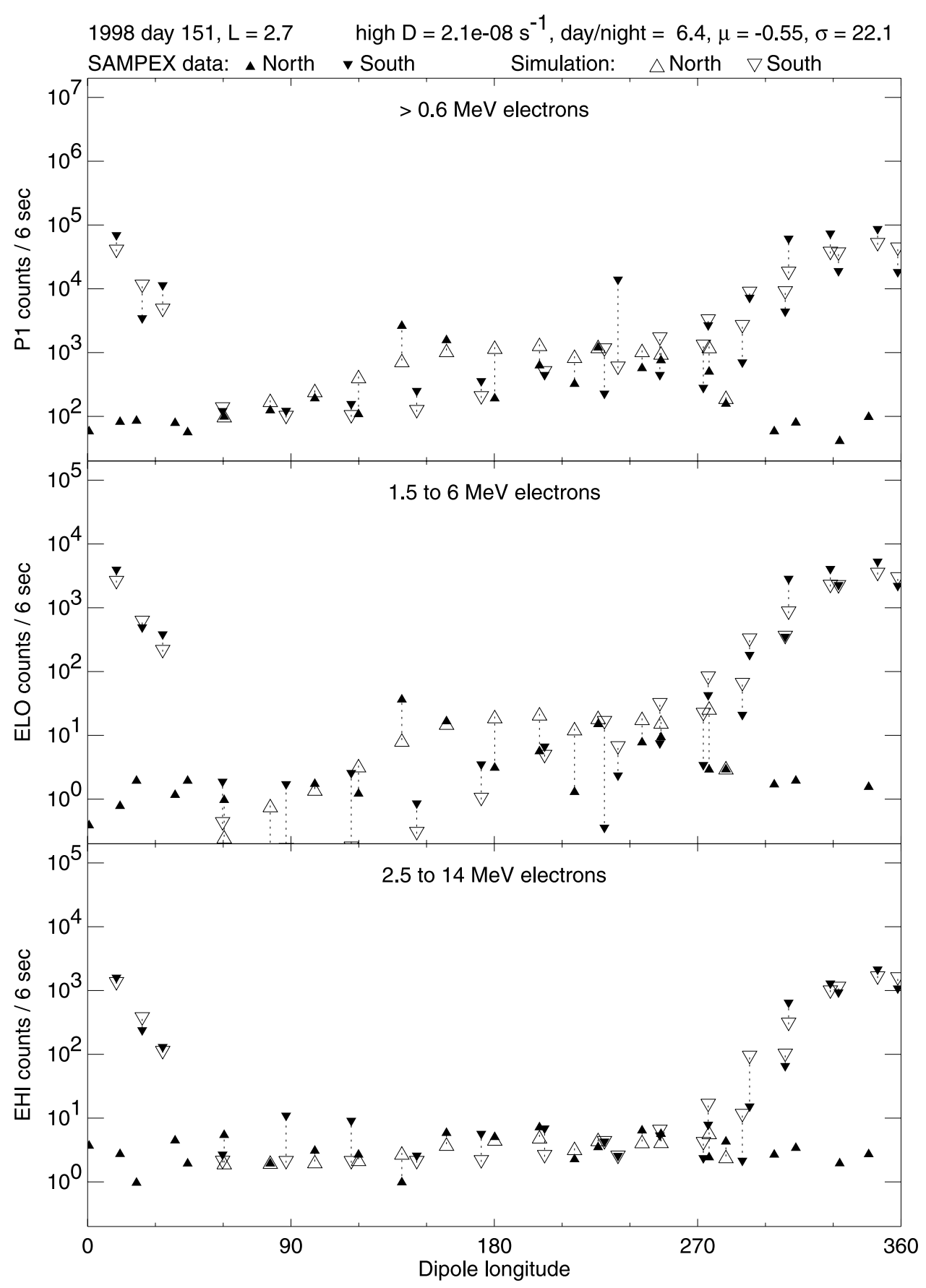

Figure 11. Similar to Figure 10 but for 1998 day 151.

(factor $\sim 10$ ) variations can also be caused by differences in either the SAMPEX pointing direction or the equatorial pitch angle of locally mirroring electrons.

[23] To further emphasize the strong day-night asymmetry apparent in the data from 1998 day 159, the same case is shown in Figure 9 but without the day-night asymmetry in the model simulation. The model parameters are the same as in the previous figure except that the nightside diffusion coefficient is increased to the same value as on the dayside. Now there is a clear mismatch between the lower data points observed in the DLC region during the second half of the day and the much higher simulated values that are similar to those observed and simulated during the first half of the day. The model with the strong day-night asymmetry was clearly a much better fit to the data.

[24] The example of 1998 day 159 is also useful in illustrating the sensitivity of the data to a range of diffusion coefficients. The factor $\sim 30$ variation from day to night produced a substantially larger flux variation in the western longitudes of the DLC region. The model diffusion coefficient is therefore well constrained by the data. The same would be true if there were no local time dependence and the diffusion coefficient were either low throughout or high throughout (as shown by the simulation in Figure 9).

[25] The best-fitting model for 1998 day 159 produced a reasonably accurate simulation for most of the data points, 


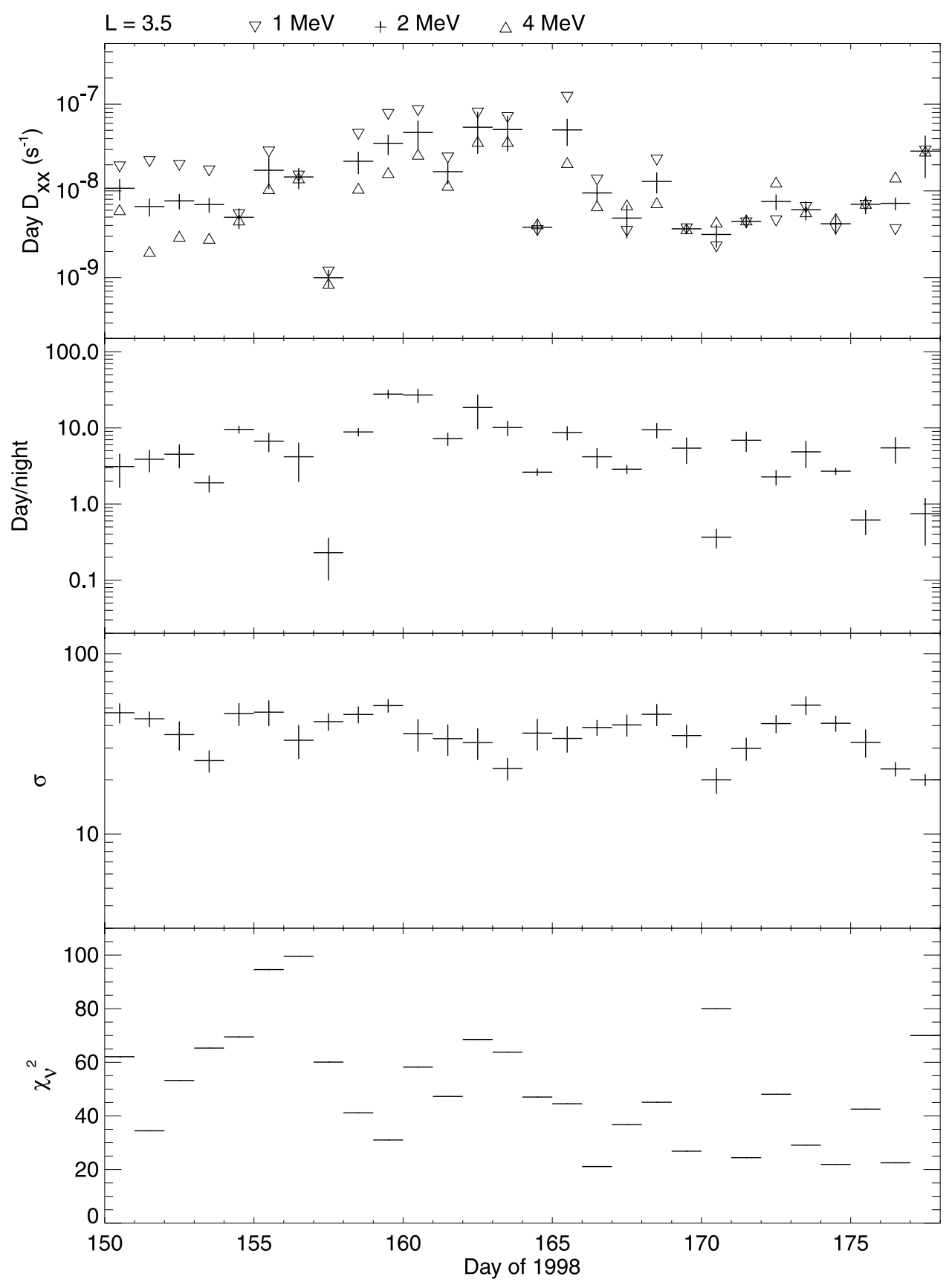

Figure 12. Model parameter estimates from 1998, $L=3.5$. Day and night diffusion coefficients are evaluated at $x=1$ and at energies of 1,2 , and $4 \mathrm{MeV}$, as indicated. Day/night is the ratio of diffusion coefficients. For clarity, error bars are shown only in the $2 \mathrm{MeV}$ case. Horizontal bars show the time range (1 day) of each fit.

considering that there are only four model parameters and $\sim 50$ data points. However, there are some systematic deviations between the simulations and the data. This is seen more clearly in Figure 10, which shows the best fit to the data from 1998 day 150 at $L=2.7$. Despite the lower $L$ shell the general nature of the data and simulation are similar to those of Figure 8. As in that case, and in other similar cases, a subset of the data is substantially below the model simulations. These points occurred at similar times ( $\sim 2300$ UT) and longitudes $\left(\sim 180^{\circ}\right.$ to $\left.270^{\circ}\right)$ on each day, suggestive of systematic errors in the model. For example, assuming a greater local time extent of the low diffusion region on the nightside or a more gradual transition from the day to night diffusion coefficients may improve the fits.

[26] A similar effect on the simulated data to that of the day-night asymmetry can be obtained by assuming that the diffusion coefficient has no local time asymmetry but varies with UT, being higher in the first 12 UT hours of the day than in the second 12 UT hours (this is evident from the similarity of Figure 1a with Figure 1d and Figure 1c with Figure 1f). In some cases, usually those showing significant systematic errors with the day-night asymmetry model such 


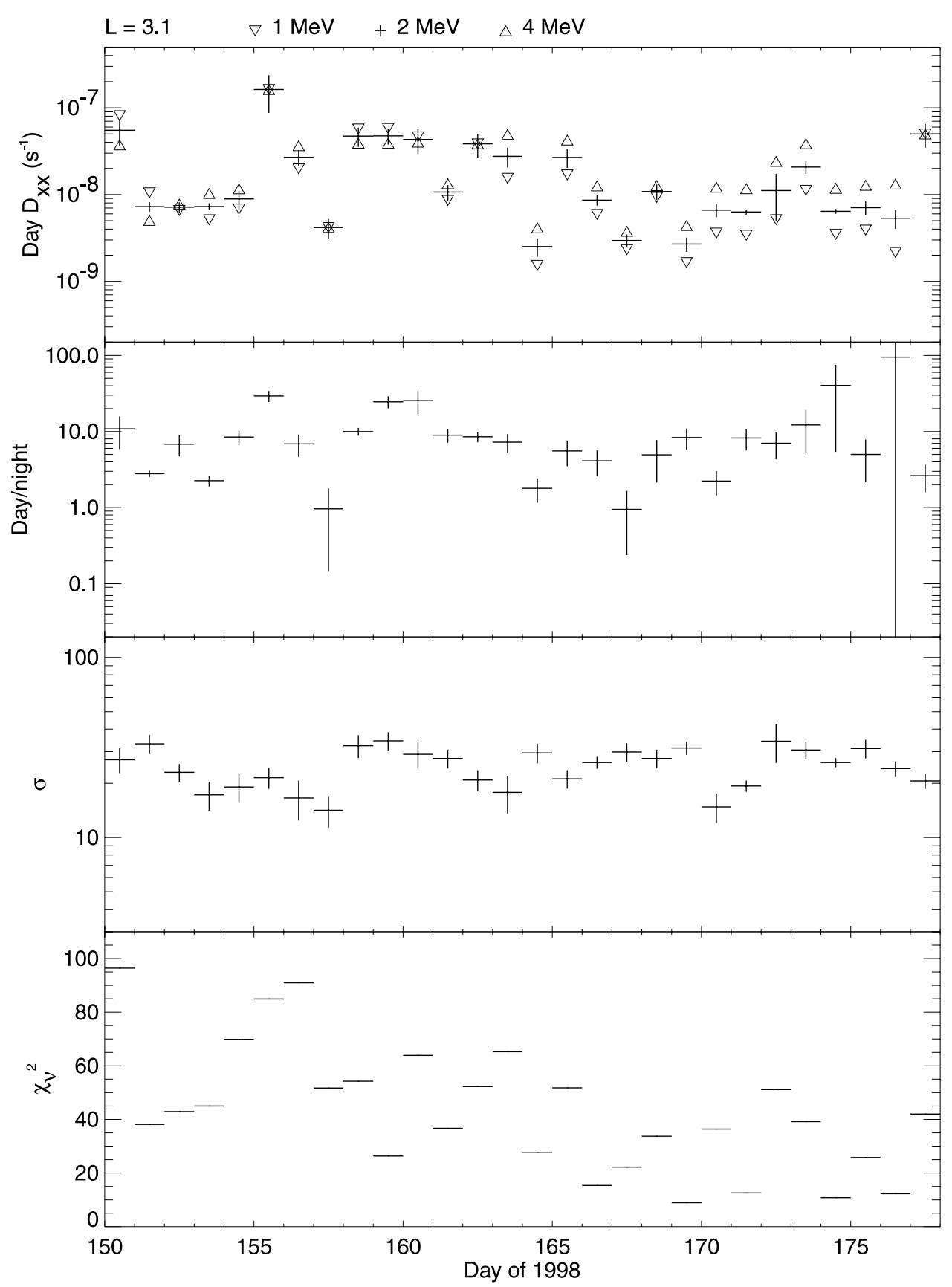

Figure 13. Model parameter estimates from 1998, $L=3.1$, as in Figure 12 .

as on day 150 (Figure 10), the fit can be slightly improved by using this alternate model. In other cases the reverse is true. The day-night asymmetry seems preferable to a UT periodicity in the diffusion coefficient primarily because of our prior knowledge of the diffusion mechanism, as discussed further below.

[27] A final example is shown in Figure 11 from 1998 day 151 and $L=2.7$, the day after the case of Figure 10 . The stably trapped electron fluxes (near 0 and $360^{\circ}$ dipole longitude) are almost unchanged from the previous day, but the data and simulations in the DLC region $\left(\sim 60^{\circ}\right.$ to $300^{\circ}$ ) are substantially lower. In the simulations, this is a consequence of a significant decrease in the diffusion coefficients, both at day and night, as shown in the model parameters. This must be a true decrease rather than modeling error because it occurs in the data taken throughout the day. While the change in $D_{x x}$ occurred near the boundary of days 150 and 151 , it is likely that such variations can also occur at other times during a day, causing some mismatches between the data and simulations.

\section{Parameter Estimates}

[28] To find the model parameters that best fit each 1-day subset of the SAMPEX data, we minimize the function $\chi^{2}=$ $\Sigma_{i}\left(y_{i}-\tilde{y}_{i}\right)^{2} / \sigma_{i}^{2}$ where $y_{i}=\ln r_{i}, r_{i}$ is the observed flux, $\tilde{y}_{i}$ is the simulated value of $y_{i}$, and $\sigma_{i}$ (unrelated to $\sigma$ in equation (2)) is the standard uncertainty in $y_{i}$. For $\sigma_{i}$ we 


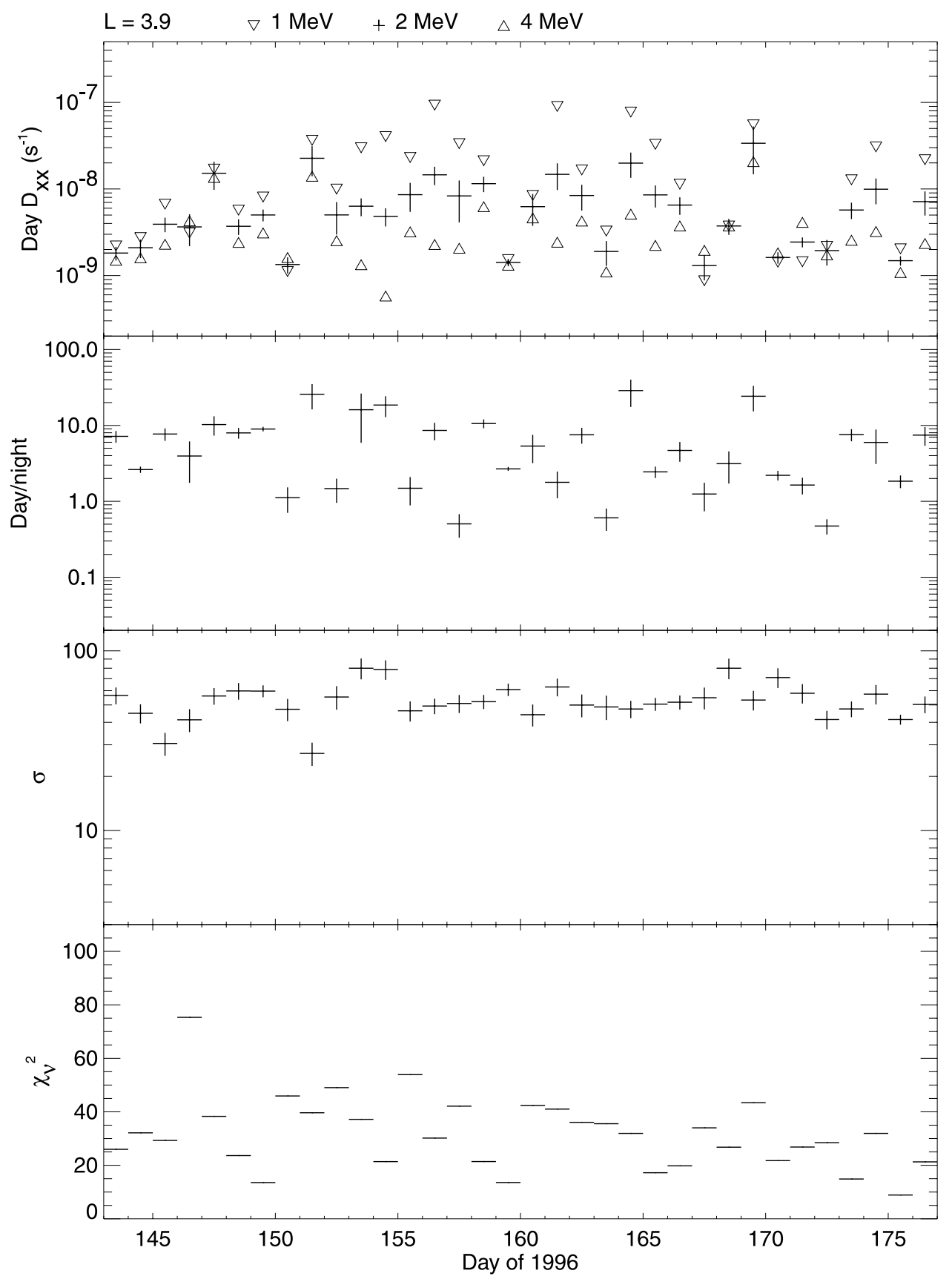

Figure 14. Model parameter estimates from 1996, $L=3.9$, as in Figure 12 .

include uncertainties due to Poisson counting statistics, which are significant for the low fluxes, and an additional $10 \%$ modeling uncertainty to prevent the fit from being constrained by only the high flux points. This can include the small uncertainties $(\sim 5 \%)$ in the instrumental response functions (Appendix A) because they lead to systematic relative errors in the simulation of the same type as any modeling errors. However, the assumed uncertainties are meant only to provide reasonable weighting factors to the data and clearly do not account for the actual mismatch from the simulation (e.g., Figure 10). Uncertainties in the final model parameters are estimated by the standard leastsquares techniques, but to account for the systematic deviations of the model from the data, at least in an approximate way, the parameter variances are multiplied by the reduced $\chi^{2}$, that is by $\chi_{v}^{2}=\chi^{2} / v$ where $v$ is the number of degrees of freedom (number of data points minus number of fit parameters). The fit parameters were allowed to vary in the ranges of $5 \times 10^{-10}$ to $1 \times 10^{-6} \mathrm{~s}^{-1}$ for the larger of $D_{\text {day }}$ and $D_{\text {night }}, 0.01$ to 100 for the day/night ratio, -5 to 5 for $\mu$, and 10 to 80 for $\sigma$.

[29] Examples of model fits were shown in the previous section. Results from the full 1998 time period and $L=3.5$ are shown in Figure 12. The dayside diffusion coefficients for electron energies of $E=1,2$, and $4 \mathrm{MeV}$ and for $x=1$ are calculated from the estimated parameters by $D_{x x}=$ $D_{d a y} E^{-\mu}$. The $2 \mathrm{MeV}$ values are generally the best constrained by the particular response functions of the three 
Table 1. Summary of Estimated Model Parameters ${ }^{\mathrm{a}}$

\begin{tabular}{|c|c|c|c|c|}
\hline \multirow[b]{2}{*}{$L$ shell } & \multicolumn{2}{|c|}{$D_{x x}\left(\times 10^{-9} \mathrm{~s}^{-1}\right)^{\mathrm{b}}$} & \multirow[b]{2}{*}{$\mu$} & \multirow[b]{2}{*}{$\sigma$} \\
\hline & Day & Night & & \\
\hline \multicolumn{5}{|c|}{1996 days $143-176$} \\
\hline 3.5 & $4.3 \pm 3.3$ & $1.9 \pm 2.7$ & $0.4 \pm 0.5$ & $40 \pm 9$ \\
\hline 3.9 & $7.5 \pm 7.1$ & $2.1 \pm 3.1$ & $-0.9 \pm 1.0$ & $53 \pm 12$ \\
\hline \multicolumn{5}{|c|}{1997 days $180-219$} \\
\hline 3.1 & $16 \pm 14$ & $6.4 \pm 5.3$ & $1.7 \pm 0.9$ & $25 \pm 9$ \\
\hline 3.5 & $9.1 \pm 10$. & $2.9 \pm 3.1$ & $0.2 \pm 0.8$ & $43 \pm 12$ \\
\hline 3.9 & $7.4 \pm 8.3$ & $3.0 \pm 4.2$ & $-0.6 \pm 0.6$ & $61 \pm 15$ \\
\hline \multicolumn{5}{|c|}{1998 days $150-177$} \\
\hline 2.7 & $58 \pm 120$ & $3.6 \pm 3.1$ & $0.3 \pm 0.8$ & $19 \pm 4$ \\
\hline 3.1 & $24 \pm 32$ & $3.1 \pm 3.5$ & $0.4 \pm 0.5$ & $25 \pm 6$ \\
\hline 3.5 & $16 \pm 16$ & $4.2 \pm 7.2$ & $-0.4 \pm 0.7$ & $37 \pm 9$ \\
\hline 3.9 & $15 \pm 16$ & $3.3 \pm 5.6$ & $-1.5 \pm 0.8$ & $47 \pm 9$ \\
\hline
\end{tabular}

${ }^{\mathrm{a}}$ Mean and standard deviation of daily values in each period.

${ }^{\mathrm{b}}$ Evaluated at $E=2 \mathrm{MeV}$ and $x=1$.

PET rate counters. The 1 and $4 \mathrm{MeV}$ uncertainties are therefore somewhat higher than for the $2 \mathrm{MeV}$ case but, for clarity, only the uncertainties at $2 \mathrm{MeV}$ are shown in the figure. The day/night ratio is simply $D_{d a y} / D_{\text {night }}$ and neither it nor $\sigma$ (the parameter for the $x$ dependence) are functions of energy. The uncertainties shown for $\sigma$ are probably larger than necessary because that parameter is constrained primarily by the stably trapped electrons near the SAA that are less influenced by the systematic modeling errors in the DLC region. The variations of electron flux with longitude in the DLC region are the primary constraints on both the dayside diffusion coefficient and the day/night ratio. As described above, there are variations in the estimated parameters from day to day, but there are also trends that extend over periods of several days. The relative quality of fits can be judged from the values of $\chi_{v}^{2}$ also shown in the figure, but the general trend of decreasing $\chi_{v}^{2}$ is a result of the gradually decreasing trapped electron flux relative to the fixed background levels and does not indicate improving fit quality.

[30] Estimated parameters from 1998, $L=3.1$ are shown in Figure 13. The trends are similar to those at $L=3.5$ (Figure 12), with some systematic differences. The corresponding results from 1996, $L=3.9$ are shown in Figure 14. Rather than including all of the available results, a summary is given in Table 1 . For each of the three time periods the means and standard deviations of the estimated $D_{x x}$ (at $E=2 \mathrm{MeV}$ and $x=1$ ), $\mu$ and $\sigma$ values are listed. The standard deviations of the daily estimates are included, rather then the uncertainties in the means (which would be much smaller), in order to approximate the degree of variability in each case. The lower $L$ values are not available for the 1996 and 1997 periods because the Polar satellite orbit did not reach low $L$ at those times.

[31] The estimated model parameters can be used to derive $e$-folding lifetimes, as in Figure 3 , based on the mean of the day and night diffusion coefficient from each fit. These are shown for $L=3.5$ and each of the time intervals in Figure 15. The geomagnetic index $D_{s t}$ is also shown in the figure for comparison with the variations in lifetime. To show correlations more clearly, the $E=2 \mathrm{MeV}$ lifetimes are shown versus $D_{s t}$ for the four selected $L$ shells and all three time periods combined (where available) in Figure 16. This figure also provides a summary of all of the lifetime results from the three time periods.

\section{Discussion}

[32] We have shown that a combination of azimuthal drift and pitch angle diffusion into the upper atmosphere describes the essential features of low-altitude radiation belt energetic electron data during times of steady decay. The loss rate is determined by the rate of diffusion, but drift must also be included to account for the observed azimuthal distribution of electrons in the drift loss cone and that distribution constrains the value of the diffusion coefficient.

[33] The model was also constrained by data at high altitudes that provided the upper boundary condition for the model simulations. The high-altitude data are not essential because the electron intensity there could be an additional free parameter, but their inclusion provides a much stronger constraint on the equatorial pitch angle $\left(\alpha_{0}\right)$ dependence of the diffusion coefficient than would the low-altitude data alone, while the agreement of the model with both data sets increases our confidence in the final results.

[34] The low-altitude data provided most of the critical information for determining the diffusive loss rates because they were obtained within and just above the electron drift loss cone (DLC), that is the region where the balance between drift and diffusion determines the longitudinal electron distribution. It is also only at low altitude that the satellite orbital period is short enough to provide good longitudinal coverage on a daily time scale. It was critical to the accuracy of the final results to include accurate and detailed information on the angular and energy dependencies of the electron detectors as well as the satellite orbital and pointing data.

[35] The solutions of the drift-diffusion model used to fit the data are applicable to steady electron decay. Transient effects of varying magnetospheric conditions were not included. However, the resulting parameter estimates in fact did show significant variability in the diffusion coefficient on a daily time scale. For a diffusion coefficient of the form $D_{x x}=D x^{-\sigma}\left(x=\cos \alpha_{0}\right)$, a change in the coefficient $D$ causes a change in the diffusion rate and the longitudinal electron distribution in the DLC but not in the shape of the high-altitude pitch angle distribution. Therefore the electron distribution can readjust to the change in $D$ on a time scale of only one drift period or $\sim 10$ to $50 \mathrm{~min}$ for an electron energy range of 5 to $0.5 \mathrm{MeV}$. Daily variations in the fit parameters relating to the diffusion rate, that is $D_{\text {day }}$, $D_{\text {night }}$, and $\mu$ in the notation of our model, can therefore be accommodated by steady decay solutions. The same is true for the daily variation at a fixed longitude caused by any day-night asymmetry in $D_{x x}$.

[36] Variations in $\sigma$, which provides the model $x$ dependence, require longer time scales for the pitch angle distribution to adjust to its new steady decay state. Time scales on the order of the electron lifetimes would be required for significant changes. Therefore changes in the estimated $\sigma$ values should be considered reliable only on time scales of several days or more. However, since we are concerned 

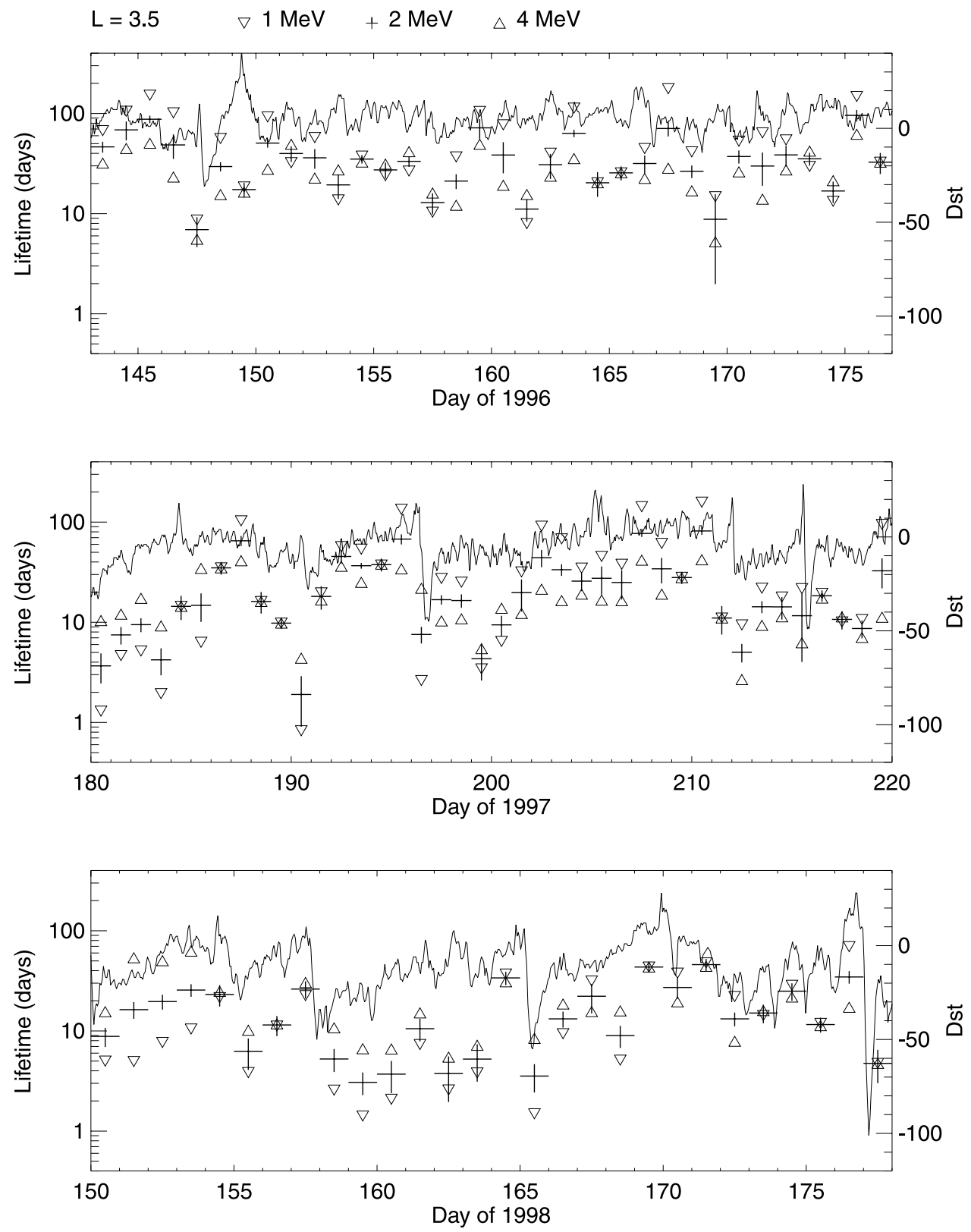

Figure 15. The $e$-folding lifetimes (data points), derived from the model parameter estimates for three time periods at $L=3.5$, and the $D_{s t}$ geomagnetic index.

primarily with the diffusive loss rates obtained from the low-altitude electron distributions, this is not a significant shortcoming of our analysis.

[37] The model included a day-night asymmetry in the diffusion coefficient. This was required to fit 1-day subsets of the data, although it would also be possible to use a daily periodicity in the time dependence of the diffusion coefficient rather than the local time dependence. The data required significantly faster diffusion on the dayside than on the nightside for most of the time. Occasionally, the nightside diffusion was faster. On the average (Table 1), the day/night ratio was $\sim 2$ to 3 during the 1996 and 1997 periods but was $\sim 4$ to 20 during the 1998 period (being higher at lower $L$ ) due to higher dayside diffusion. Daily variations in the day-night ratio were comparable to the ratio itself, but large changes tended to be isolated rather than randomly distributed. The higher diffusion rates in daytime compared with nighttime appear to be consistent with observations [Russell et al., 1969; André et al., 2002] of a similar local time distribution in the plasmaspheric hiss. It is the ELF electromagnetic emission thought to be the primary cause of electron pitch angle scattering within the part of the outer zone radiation belt that is inside the plasmasphere ( $L \sim 2.5$ to 4) [Abel and Thorne, 1998].

[38] The estimated values of the diffusion coefficient correspond to electron diffusive loss lifetimes (averaged over day and night) that vary over a wide range. Given the uncertainties in the estimates, the lifetimes are well correlated with the $D_{s t}$ geomagnetic index (Figure 16), the shorter lifetimes generally corresponding to higher geomagnetic activity. The average activity level was lowest during 

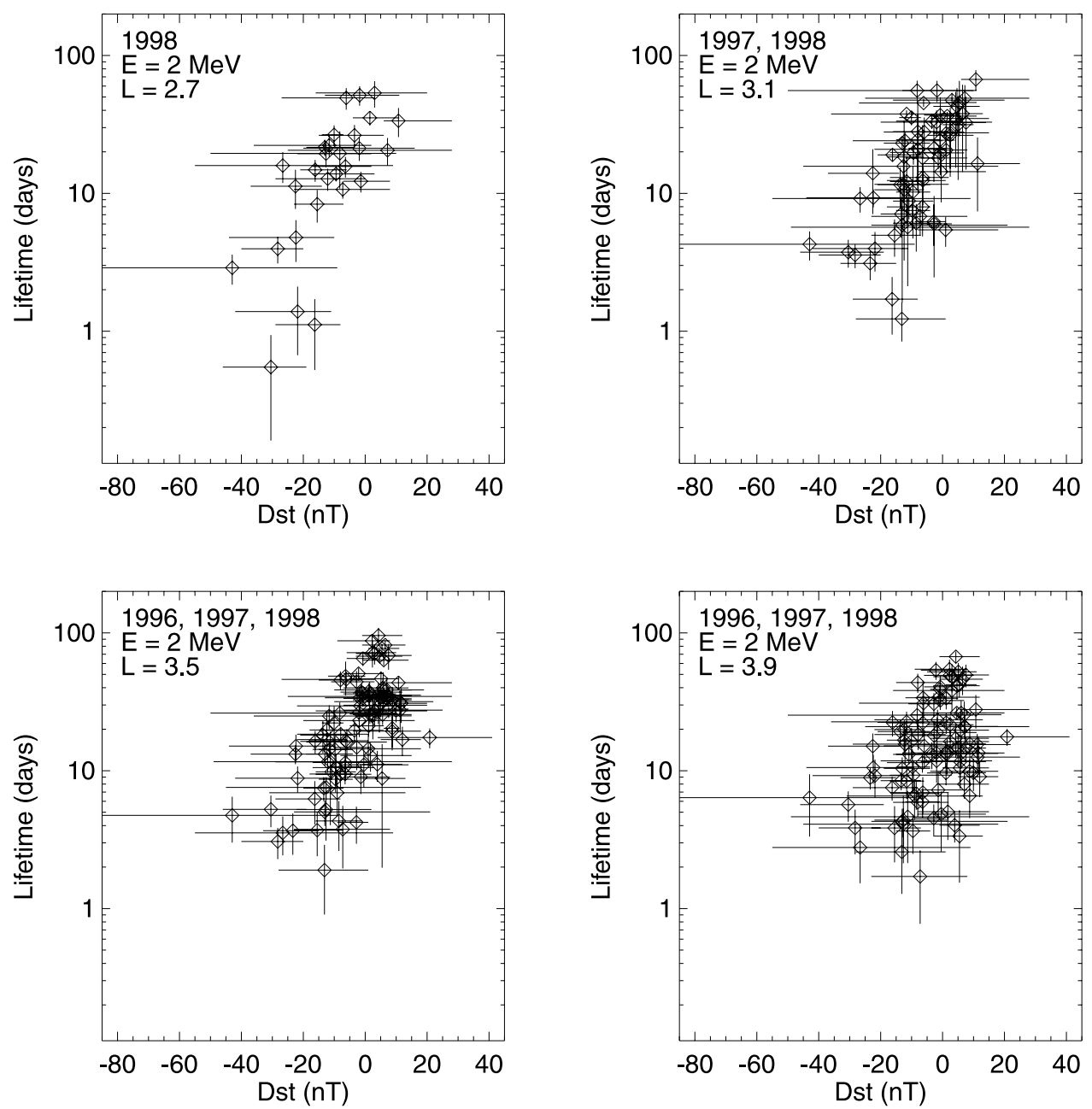

Figure 16. Derived $e$-folding lifetimes at $E=2 \mathrm{MeV}$ versus the $D_{s t}$ geomagnetic index, for four selected $L$ shells. The lifetimes are for 1-day periods and points are plotted at the mean $D_{s t}$ value for the day. The error bars on the lifetimes are derived from the data fits, and those on $D_{s t}$ indicate the minimum and maximum value during the day.

the 1996 period and highest during the 1998 period, leading to generally weaker and stronger diffusion rates respectively, but the correlation was also evident with the specific small magnetic storms that occurred during the 1997 and 1998 periods (Figure 15). The correlation is consistent with the interpretation that scattering by plasmaspheric hiss is the principal diffusion mechanism because the hiss intensity increases during the recovery phase of geomagnetic storms [Smith et al., 1974].

[39] Observed trapped electron decay rates of $\sim 10$ to 20 days (Figure 5) are in reasonable agreement with average estimated lifetimes. However, the variability in the estimated lifetimes (Figures 15 and 16) is greater than that of the observed decay rates. The discrepancy is significant for only the occasional isolated short lifetimes, $\lesssim 1$ day, that occur primarily at the lower $L$ shells because in those instances there should be significant reductions of the trapped electron intensity during a single day. Such reductions are not observed in either the high-altitude Polar data or in the trapped component of the low-altitude SAMPEX data. The clearest example is 1998 day 158 at $L=2.7$ (Figure 10), for which the estimated lifetime at $2 \mathrm{MeV}$ is 0.55 days. Then the stably trapped intensity should decrease by a factor of $\sim 6$ during that day, but in fact it is not greatly reduced a day later (Figure 11). A possible explanation is that the trapped electrons are replenished during times of rapid losses into the atmosphere by either radial diffusion or local acceleration.

[40] The estimated values of $\sigma$ showed that the diffusion coefficient increases rapidly with decreasing $x$, or increasing mirror point altitude, in the low-altitude region. This is consistent with the measured pitch angle distributions that are flat at intermediate pitch angles and decrease rapidly near the loss cones (compare Figures 2 and 6), the flat section being due to relatively fast diffusion at high altitudes.

[41] The $\sigma$ values generally increase with $L$ (Table 1). This may coincide with a greater relative contribution to electron scattering from lightning generated whistlers at lower $L$, compared with plasmaspheric hiss at higher $L$ [Abel and Thorne, 1998; Blake et al., 2001], or some other spatial variation in the diffusion mechanism. However, we should also note that because $\sigma$ is constrained primarily by the ratio of the trapped electron fluxes measured on the low- 

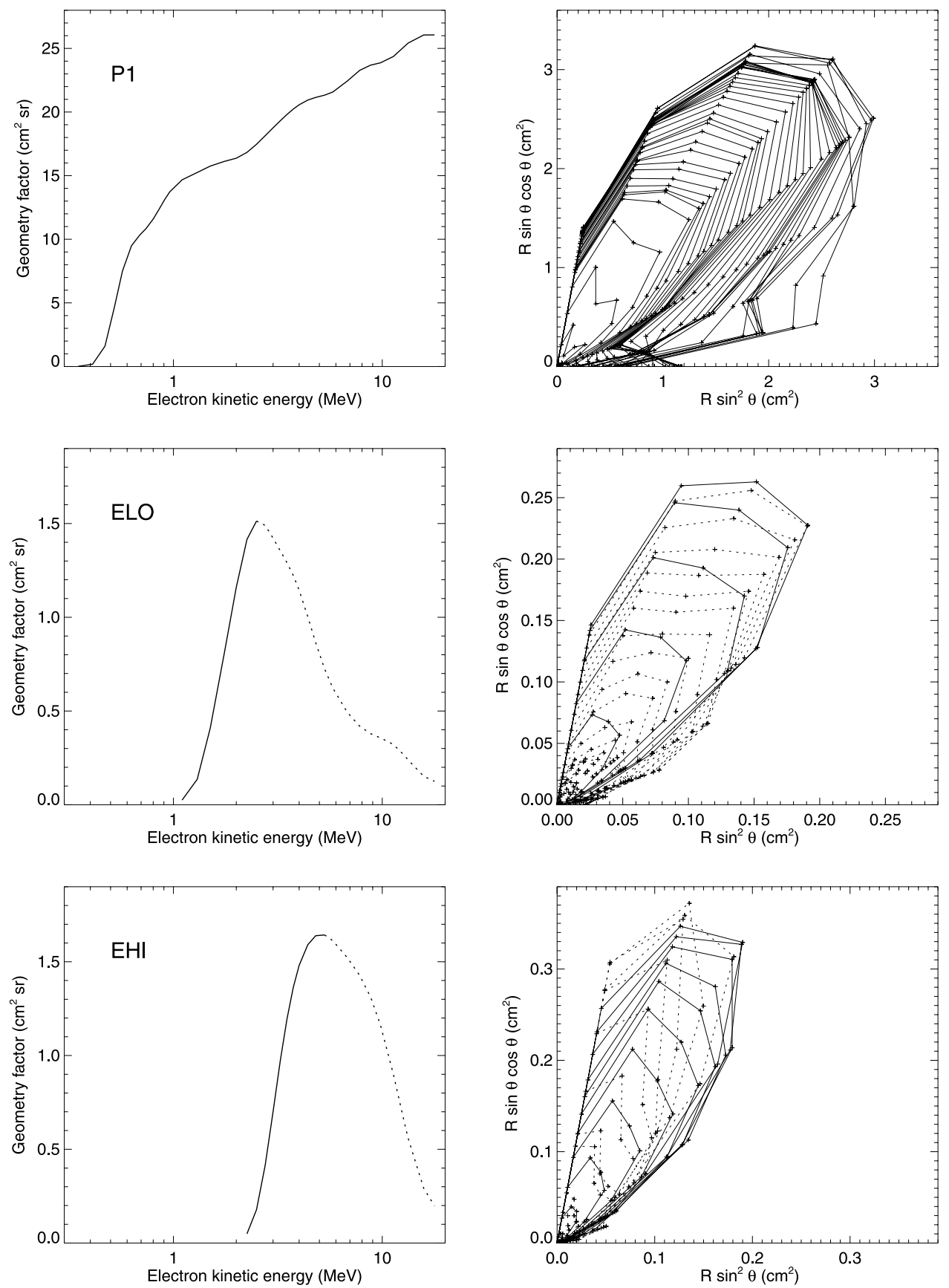

Figure A1. (left) Calibrated PET energy response curves integrated over solid angle, or geometry factors, for each of the three electron rate counters. (right) Polar plots of the corresponding angular response $R \sin \theta$, where $\theta$ is the angle from the telescope axis, at selected energies. Solid (dashed) curves are used where the response is increasing (decreasing) with energy.

altitude and high-altitude satellites, its value is sensitive to any errors in this comparison. The same is not true of the other model parameters that are constrained primarily by the low-altitude longitudinal electron distribution.

[42] The energy dependence of the diffusion coefficient was modeled only in a crude way because of the limited constraints available from the broad energy response of the three PET electron counters. Also, the accuracy of the energy dependence can be compromised by systematic modeling errors and by the assumptions that $\sigma$ and the day-night ratio are not energy dependent. However, the estimated $\mu$ values $\left(D_{x x} \sim E^{\mu}\right)$ do show some potentially significant trends. The mean values are generally positive ( $D_{x x}$ increasing with energy) at the lower $L$ values and negative ( $D_{x x}$ decreasing with energy) at the higher $L$ values, with significant daily variations. The diffusion coefficient is best constrained by the data near $E=2 \mathrm{MeV}$.

[43] It is possible that the results described above could be improved by more detailed observations, particularly in the energy dependence of the low-altitude data. However, the main source of uncertainty currently is in the model. At the expense of added complexity there are several areas 
of possible improvement: time-dependent solutions could be used, rather than the steady decay approximation; the local time dependence of the diffusion coefficient could be more realistic, possibly guided by plasma wave data; finally, the model could include other physical processes, such as atmospheric scattering with diffusion into the bounce loss cone, radial diffusion, and local acceleration.

\section{Appendix A: PET Response}

[44] The rate $r$ measured by any of the PET counters, P1, ELO, or EHI [Cook et al., 1993], is related to the local electron intensity $j$ and the counter response function $R$ by

$$
r=\int_{0}^{2 \pi} \int_{0}^{\pi} \int_{0}^{\infty} R\left(E, \theta_{t}\right) j(E, \alpha) d E \sin \theta_{t} d \theta_{t} d \phi_{t}
$$

where $\theta_{t}$ and $\phi_{t}$ are the spherical polar and azimuthal angles, respectively, relative to the telescope axis, and $\alpha$ is the local pitch angle that is related to the angle $\theta_{B}$ between the telescope axis and the local magnetic field direction by

$$
\cos \alpha=\sin \theta_{B} \sin \theta_{t} \cos \phi_{t}+\cos \theta_{B} \cos \theta_{t}
$$

The response functions were determined experimentally prior to the SAMPEX launch. For energies from 0.3 to $3 \mathrm{MeV}$, this was done using a $\mathrm{Ru}^{106}$ radioactive source and a magnet $\beta$-spectrometer at the California Institute of Technology. For energies from 1.5 to $27 \mathrm{MeV}$, the Department of Energy linear electron accelerator operated by EG\&G in Santa Barbara, California, was used. The calibration results are shown in Figure A1. The energy response curves (left side of figure) can be characterized by the approximate energy ranges of $>0.6 \mathrm{MeV}$ for $\mathrm{P} 1,1.5$ to $6 \mathrm{MeV}$ for $\mathrm{ELO}$, and 2.5 to $14 \mathrm{MeV}$ for $\mathrm{EHI}$, but the actual energy range of a given measurement depends on the spectrum. The angular response (right side of figure) also varies somewhat with energy and between rate counters. The complete calibrated response functions from Figure A1 were combined with the model intensity $j$ by numerical evaluation of equation (A1) to simulate the PET data.

[45] There are some additional corrections that must be made to the PET rates. Instrumental deadtime, which is significant at high counting rates, is measured by PET on board the satellite, and we make a correction for it in the rate data. The single detector P1 data have a significant background due to penetrating cosmic rays, while the multidetector ELO and EHI data have small backgrounds. We have added empirically determined background values of $80,0.1$, and 2 counts per $6 \mathrm{~s}$ interval to the simulated P1, ELO, and EHI data, respectively. The ELO counter requires coincident measurements in each of the P1 and P2 detectors. At high rates the ELO data include a significant fraction of chance coincidences, due to separate low-energy electrons arriving in the $\mathrm{P} 1$ and $\mathrm{P} 2$ detectors within the resolving time of the counter, $\tau \approx 2.25 \mu \mathrm{s}$. The rate of chance coincidences, which we add to the simulated ELO rate, is [Knoll, 1989, p. 636] $2 \tau r_{P 1} r_{P 2}$, where $r_{P 1}$, and $r_{P 2}$ are the P1 and $\mathrm{P} 2$ rates determined from their respective response functions (the $\mathrm{P} 2$ response function, not shown in Figure A1, was also determined by calibrations). It was not necessary to correct for chance coincidences in EHI. We have also not made any correction for pileup of low-energy electrons in the P1 measurement because that effect is significant only for relatively soft spectra.

[46] Acknowledgments. We thank two anonymous referees for valuable comments on an earlier version of this manuscript. This work was supported by NASA under grant NAG5-12766.

[47] Arthur Richmond thanks Michael Schulz and another reviewer for their assistance in evaluating this paper.

\section{References}

Abel, B., and R. M. Thorne, Electron scattering loss in the Earth's inner magnetosphere: 1. Dominant physical processes, J. Geophys. Res., 103, 2385-2396, 1998

Abel, B., and R. M. Thorne, Modeling energetic electron precipitation near the South Atlantic anomaly, J. Geophys. Res., 104, 7037-7044, 1999.

André, R., F. Lefeuvre, F. Simonet, and U. S. Inan, A first approach to model the low frequency wave activity in the plasmasphere, Ann. Geophys., 20, 981-986, 2002.

Barton, C. E., International geomagnetic reference field: The seventh generation, J. Geomagn. Geoelectr., 49, 123-148, 1997.

Blake, J. B., et al., CEPPAD: Comprehensive energetic particle and pitch angle distribution experiment on POLAR, Space Sci. Rev., 71, 531-562, 1995.

Blake, J. B., U. S. Inan, M. Walt, T. F. Bell, J. Bortnik, D. L. Chenette, and H. J. Christian, Lightning-induced energetic electron enhancements in the drift loss cone, J. Geophys. Res., 106, 29,733-29,744, 2001.

Cook, W. R., et al., PET: A proton/electron telescope for studies of magnetospheric, solar, and galactic particles, IEEE Trans. Geosci. Remote Sens., 31, 565, 1993.

Davidson, G. T., An improved empirical description of the bounce motion of trapped particles, J. Geophys. Res., 81, 4029-4030, 1976.

Imhof, W. L., Electron precipitation in the radiation belts, J. Geophys. Res., 73, 4167-4184, 1968.

Knoll, G. F., Radiation Detection and Measurement, 2nd ed., 754 pp., John Wiley, New York, 1989.

Lyons, L. R., R. M. Thorne, and C. F. Kennel, Pitch-angle diffusion of radiation belt electrons within the plasmasphere, J. Geophys. Res., 77, 3455-3474, 1972.

Pesnell, W. D., R. A. Goldberg, D. L. Chenette, E. E. Gaines, and M. Schulz, Growth and decay of relativistic electrons during a magnetic storm as seen in low-Earth orbit, J. Geophys. Res., 106, 30,039-30,046, 2001.

Roberts, C. S., Pitch angle diffusion of electrons in the magnetosphere, Rev. Geophys. Res., 7, 305, 1969.

Russell, C. T., R. E. Holzer, and E. J. Smith, OGO 3 observations of ELF noise in the magnetosphere: 1 . Spatial extent and frequency of occurrence, J. Geophys. Res., 74, 755, 1969.

Schulz, M., and L. J. Lanzerotti, Particle Diffusion in the Radiation Belts, 215 pp., Springer-Verlag, New York, 1974.

Selesnick, R. S., and J. B. Blake, Radiation belt electron observations following the January 1997 magnetic cloud event, Geophys. Res. Let., 25, 2553-2556, 1998.

Selesnick, R. S., and J. B. Blake, On the source location of radiation belt relativistic electrons, J. Geophys. Res., 105, 2607-2624, 2000.

Sheldon, W. R., On the precipitation of relativistic electrons from the outer belt, J. Atmos. Terr. Phys., 53, 17-23, 1991.

Smith, E. J., A. M. A. Frandsen, B. T. Tsurutani, R. M. Thorne, and K. W. Chan, Plasmaspheric hiss intensity variations during magnetic storms, J. Geophys. Res., 79, 2507-2510, 1974.

Williams, D. J., J. F. Arenas, and L. J. Lanzerotti, Observations of trapped electrons at low and high altitudes, J. Geophys. Res., 73, 5673, 1968.

R. S. Selesnick and J. B. Blake, The Aerospace Corporation, P.O. Box 92957-M2/259, Los Angeles, CA 90009-2957, USA. (richard.s.selesnick@) aero.org; jbernard.blake@aero.org)

R. A. Mewaldt, 220-47 Caltech, Pasadena, CA 91125, USA. (rmewaldt@) srl.caltech.edu) 